\title{
Coupled rotational dynamics of Saturn's thermosphere and magnetosphere: a thermospheric modelling study
}

\author{
C. G. A. Smith ${ }^{1, *}$ and A. D. Aylward ${ }^{1}$ \\ ${ }^{1}$ Atmospheric Physics Laboratory, Department of Physics and Astronomy, University College, London, UK \\ *now at: Teesdale School, Barnard Castle, County Durham, UK
}

Received: 2 April 2007 - Revised: 3 December 2007 - Accepted: 17 December 2007 - Published: 13 May 2008

\begin{abstract}
We use a numerical model of Saturn's thermosphere to investigate the flow of angular momentum from the atmosphere to the magnetosphere. The thermosphere model is driven by Joule heating and ion drag calculated from a simple model of the magnetospheric plasma flows and a fixed model of the ionospheric conductivity. We describe an initial study in which our plasma flow model is fixed and find that this leads to several inconsistencies in our results. We thus describe an improved model in which the plasma flows are allowed to vary in response to the structure of the thermospheric winds. Using this improved model we are able to analyse in detail the mechanism by which angular momentum extracted from the thermosphere by the magnetosphere is replaced by transport from the lower atmosphere. Previously, this transport was believed to be dominated by vertical transport due to eddy viscosity. Our results suggest that transport within the upper atmosphere by meridional winds is a much more important mechanism. As a consequence of this, we find that the rotational structures of the thermosphere and magnetosphere are related in a more complex way than the eddy viscosity model implies. Rather than the thermosphere behaving as a passive component of the system, the thermosphere-magnetosphere interaction is shown to be a two-way process in which rotational structures develop mutually. As an example of this, we are able to show that thermospheric dynamics offer an explanation of the small degree of super-corotation that has been observed in the inner magnetosphere. These results call into question the usefulness of the effective Pedersen conductivity as a parameterisation of the neutral atmosphere. We suggest that a two-parameter model employing the true Pedersen conductivity and the true thermospheric rotation velocity may be a more accurate representation of the thermospheric behaviour.
\end{abstract}

Correspondence to: C. G. A. Smith

(cgasmith@gmail.com)
Keywords. Magnetosphere (Magnetosphere-ionosphere interactions; Planetary magnetosphere) - Meteorology and atmospheric dynamics (Thermospheric dynamics)

\section{Introduction}

Saturn's magnetosphere is dominated by the influence of the rapid $\sim 11 \mathrm{~h}$ planetary rotation frequency. This rotation frequency manifests itself in a number of different ways: in the actual physical rotation of the frozen-in plasma in the magnetosphere (Richardson, 1986; Sittler et al., 2006); in the periodicity of the planetary radio emissions (e.g. Gurnett et al., 2005); and in magnetic field measurements (e.g. Cowley et al., 2006). This paper is concerned with the first of these phenomena - the actual physical rotation of the plasma - and its relationship to the rotational dynamics of the planet's neutral upper atmosphere. A possible relationship between the latter phenomena and the dynamics of the neutral upper atmosphere has been suggested by Smith (2006a).

The physical rotation of the magnetosphere occurs because planetary angular momentum is transferred from the upper atmosphere. The key stage in this process is the exchange of energy and momentum between the ionised and neutral upper atmospheres through ion-neutral collisions. These collisions drive a system of coupling currents that acts to transfer angular momentum to the magnetosphere, as discussed by Hill (1979) in the Jovian context.

The transfer of angular momentum from the upper atmosphere to the magnetosphere necessarily slows the rotation of the upper atmosphere. In steady state the angular momentum that is extracted must continually be replaced by transport from the lower atmosphere, which acts as an essentially infinite reservoir of angular momentum. The conventional understanding of this process is that the magnetospheric drag induces a vertical velocity shear in the neutral atmosphere that causes angular momentum to be transported

Published by Copernicus Publications on behalf of the European Geosciences Union. 


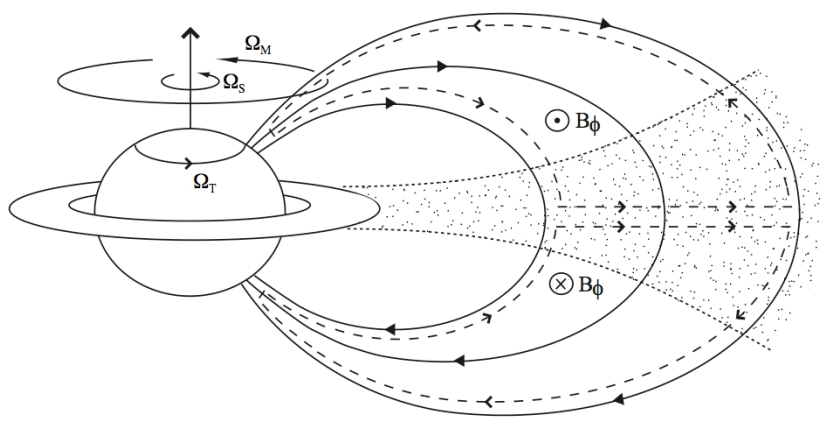

Fig. 1. Schematic diagram of Saturn's magnetosphere (adapted from Cowley and Bunce, 2003). Solid lines show the planetary magnetic field; dashed lines show the Hill current system. $\Omega_{S}, \Omega_{T}$ and $\Omega_{M}$ are the rotational velocities of the lower atmosphere, thermosphere and magnetosphere, respectively.

vertically by eddy viscosity (Huang and Hill, 1989; Pontius, 1995, 1997), maintaining partial corotation of the upper atmosphere. These studies (which refer specifically to Jupiter, but apply in principle to the Saturn case, as implied by e.g. Cowley and Bunce, 2003; Saur, 2004) explicitly neglect angular momentum transport by thermospheric winds.

More recently it has been suggested by Millward et al. (2005) that meridional and zonal thermospheric winds may have an important influence on the rotational structure of the Jovian magnetosphere. Their study, using a numerical model of the Jovian thermosphere and ionosphere (JIM), found that regions of the thermosphere close to, but not contiguous with, the main Jovian electrojet rotated more slowly than the regions of the magnetosphere to which they were connected. Millward et al. suggest that in these regions it may be that rather than the magnetosphere driving the dynamics of the thermosphere, the thermosphere may be driving the dynamics of the magnetosphere. The suggestion is tentative because the low spatial resolution of JIM, its primitive plasma flow model and the complications introduced by Jupiter's tilted magnetic dipole mean that it is difficult to analyse the model output with sufficient confidence.

This study aims to investigate the physical processes shaping the coupled dynamics of the thermosphere and magnetosphere in greater detail. We again use a numerical model, but greatly simplified in order to permit proper analysis of the model output. We also focus on Saturn rather than Jupiter. This is for two reasons: firstly because of the present research focus on Saturn stimulated by the Cassini mission; secondly because Saturn's almost axially aligned magnetic field (Davis and Smith, 1990; Dougherty et al., 2005) allows us to further simplify our model without overly compromising its realism.

Our model has already been used for baseline studies of the thermal structure of the thermosphere (Smith et al., 2005; Müller-Wodarg et al., 2006). To study the interaction with the magnetosphere the model has been adapted to include explicitly the effects of Joule heating and ion drag. Results from this model have already been presented by Smith et al. (2007), who emphasised the effect of magnetospheric forcing on the thermal structure of the upper atmosphere. Here we present further results from the same model, emphasising instead the dynamical structures and the coupling to the magnetosphere.

In Sect. 2 we outline our current understanding of the coupling between Saturn's thermosphere and magnetosphere. In Sects. 3 and 4 we then briefly describe our basic model and its output; these results motivate us to modify this basic model, and in Sects. 5 and 6 we describe these modifications and their consequences. To generalise these results we then discuss in Sect. 7 the sensitivity of our results to an increase in the ionospheric conductivity. In Sect. 8 we discuss some of the implications of our results for other studies. Finally, in Sect. 9, we conclude.

\section{Background theory}

In this section we outline briefly the normal theoretical description of the transfer of planetary angular momentum to the magnetosphere. We begin with an overview of the system of magnetosphere-atmosphere coupling currents responsible for this transfer, before then presenting a more detailed discussion of the processes taking place specifically in the upper atmosphere.

\subsection{Overview}

The current system that transfers planetary angular momentum to the magnetosphere was first described by Hill (1979) for the case of Jupiter. For Saturn, this current system is sketched in Fig. 1. Radial currents in the equatorial magnetosphere are linked by magnetic field-aligned currents to meridional currents in the upper atmosphere. In the situation shown, the currents in the equatorial magnetosphere flow away from the planet - corresponding to a $\boldsymbol{j} \times \boldsymbol{B}$ force that accelerates the plasma towards the planetary angular velocity - while the currents in the upper atmosphere flow towards the equator - corresponding to a $\boldsymbol{j} \times \boldsymbol{B}$ force that slows the rotation of the neutral upper atmosphere. The torques exerted by these $\boldsymbol{j} \times \boldsymbol{B}$ forces are equal and opposite, such that angular momentum is transferred from the upper atmosphere to the magnetosphere.

These currents are ultimately driven by the inertia of the plasma in the magnetosphere. Since the plasma is "frozen in" all the way along the magnetic field lines from the equatorial magnetosphere to the top of the ionosphere, any difference between the angular velocity of the plasma in the equatorial magnetosphere and the angular velocity of the connected neutral atmosphere manifests itself as an electric field in the rest frame of the neutral upper atmosphere. This electric field 
drives equatorwards Pedersen currents in the upper atmosphere which must close in the magnetosphere. If the inertia of the magnetosphere was negligible, it would be spun into corotation instantaneously, shutting down the current system. However, the inertia is far from negligible, and fresh inertia is supplied continually from rings and moons, such that the transfer of angular momentum is a continual and steady process. The angular momentum supplied to the magnetosphere by the planet is ultimately lost from the system by downtail outflow associated with the Vasyliūnas cycle (Vasyliūnas, 1983).

Thus angular momentum is continually extracted from the region of the neutral upper atmosphere that coexists with the ionosphere. We wish to understand this process so that we can understand the parallel process by which this angular momentum is continually replaced by transport from the lower atmosphere.

\subsection{Upper atmosphere}

For simplicity, we assume a vertical magnetic field $B$ in the ionosphere (positive upwards in the Northern Hemisphere of Saturn) and an eastwards plasma drift velocity $v_{\phi}$. Note that if the plasma drift is sub-corotational then $v_{\phi}$ is negative, and is related to the rotational velocity $\Omega_{M}$ of the connected region of the magnetosphere by

$v_{\phi}=-\left(\Omega_{S}-\Omega_{M}\right) \rho_{i}$

where $\Omega_{S}$ is the angular velocity of the planet, and $\rho_{i}$ is the perpendicular off-axis distance in the ionosphere.

This plasma drift implies a meridional electric field $E_{\theta}=-v_{\phi} B$, which is equatorwards if the plasma drift is sub-corotational. Given local Pedersen and Hall conductivities $\sigma_{P}$ and $\sigma_{H}$, their ratio $r=\sigma_{H} / \sigma_{P}$, and a neutral wind with eastwards and southwards components $u_{\phi}=k_{\phi} v_{\phi}$ and $u_{\theta}=k_{\theta} v_{\phi}$ it can easily be shown that the components of the horizontal $\boldsymbol{j} \times \boldsymbol{B}$ (ion drag) force acting on the neutrals at some altitude are:

$$
\begin{aligned}
{[\boldsymbol{j} \times \boldsymbol{B}]_{\theta} } & =\sigma_{P} B E_{\theta}\left[k_{\theta}+r\left(1-k_{\phi}\right)\right] \\
{[\boldsymbol{j} \times \boldsymbol{B}]_{\phi} } & =\sigma_{P} B E_{\theta}\left[-\left(1-k_{\phi}\right)+r k_{\theta}\right]
\end{aligned}
$$

These expressions represent forces on the neutrals due to both Pedersen and Hall currents driven by both zonal and meridional electric fields. Inclusion of the meridional winds coupled to the Hall conductivity shows that there is a component of zonal drag associated with meridional winds. This means that even if the zonal neutral and plasma velocities are equal $\left(k_{\phi}=1\right)$ there is still a drag force between the thermosphere and the plasma, provided the meridional wind and Hall conductivity are both non-zero. Thus, if we are to describe the interaction between the ions and neutrals as a simple drag force between two differentially rotating bodies, then the neutral velocity that the plasma in the magnetosphere "feels" via the atmospheric conductivity should be expressed as a linear combination of the zonal and meridional neutral velocities. We call this linear combination $k$ :

$k=k_{\phi}+r k_{\theta}$

The zonal component of the $\boldsymbol{j} \times \boldsymbol{B}$ force can now be rewritten:

$[\boldsymbol{j} \times \boldsymbol{B}]_{\phi}=-\sigma_{P} E_{\theta} B(1-k)$

When $k=1$ the zonal $\boldsymbol{j} \times \boldsymbol{B}$ force falls to zero, and $k$ thus represents an "effective" neutral velocity.

We now consider two limiting cases. Firstly, the situation that applies to the majority of the thermosphere-ionosphere, in which the Pedersen is much greater than the Hall conductivity, i.e. $r \ll 1$, such that $k \sim k_{\phi}$ :

$[\boldsymbol{j} \times \boldsymbol{B}]_{\phi} \simeq-\sigma_{P} E_{\theta} B\left(1-k_{\phi}\right)$

and this represents a simple westwards drag on the neutrals caused by the westwards drifting plasma. This approximation, in which the Hall conductivity and meridional winds are neglected, is the simple situation adopted by previous studies (Huang and Hill, 1989; Cowley et al., 2004; Millward et al., 2005; Smith et al., 2005). The opposite case is that encountered when $r \gg 1$. This corresponds to altitudes below the thermosphere where collisions are frequent enough to fully couple the ions and neutrals, and the Hall conductivity dominates:

$[\boldsymbol{j} \times \boldsymbol{B}]_{\phi} \simeq \sigma_{H} E_{\theta} B k_{\theta}=-n_{i} e B u_{\theta}$

Thus in this situation the meridional current is driven directly by ion-neutral collisions, as the neutral winds entirely control the ion motion. An equatorwards wind in this situation will have the effect of spinning up the connected magnetosphere, and spinning down the thermosphere. For this situation to persist, there must of course be a supply of angular momentum to this region of the atmosphere to balance the sub-corotational drag. We leave as an open question whether sufficient electron density exists in the Hall conducting region for such effects to be important to the structure of either the atmosphere or the magnetosphere; for further discussion see Smith (2006a).

For completeness, we note that the total energy inputs from the magnetosphere to the thermosphere due to Joule heating and ion drag are:

$q_{\mathrm{tot}}=\sigma_{P} E_{\theta}^{2}(1-k)$

This paper is not directly concerned with energy inputs, so we will not discuss this expression further here. For further discussion of the energy inputs and their consequences see Smith (2006b) and Smith et al. (2007).

All of the quantities discussed above represent local values in the thermosphere: forces are per unit volume and current densities are per unit area. Most magnetospheric studies (e.g. Cowley and Bunce, 2003) explicitly or implicitly treat 
the conducting layer of the upper atmosphere as a thin sheet, describing height-integrated forces per unit area and heightintegrated current densities per unit length. It can then be shown easily that the height-integrated $\boldsymbol{J} \times \boldsymbol{B}$ force is:

$[\boldsymbol{J} \times \boldsymbol{B}]_{\phi}=-\Sigma_{P}(1-K) E_{\theta} B$

where

$\Sigma_{P}=\int \sigma_{P} d z$

is the height-integrated Pedersen conductivity and

$\Sigma_{P} K=\int \sigma_{P} k d z$

where $K$ is now the height-integrated analogue of the neutral wind parameter $k$, incorporating contributions from zonal and meridional winds. This implies an "effective rotation velocity" of the neutral atmosphere, $\Omega_{T}$ :

$\Omega_{S}-\Omega_{T}=K\left(\Omega_{S}-\Omega_{M}\right)$

Here, the expression on the LHS is the "effective corotation lag" of the neutrals: this is equal to a fraction $K$ of the "corotation lag" of the plasma. If there is a set of circumstances across which $K$ is approximately constant then there is a linear relationship between the corotation lag of the plasma and that of the neutrals. (Note that what we call " $K$ " is referred to as " $k$ " by Cowley and Bunce, 2003, and " $1-\alpha$ " by Huang and Hill, 1989.)

The conventional physical interpretation of $K$ is derived from studies by Huang and Hill (1989) and Pontius (1995), in the Jovian context. Assuming that the sub-corotational torque imposed on the thermosphere by the magnetosphere is balanced by upwards viscous transfer of angular momentum, they showed that $K$ is dependent on the atmospheric structure, but that for a given atmospheric structure it is independent of the plasma velocity. Thus the "slippage" of the neutral atmosphere in response to the magnetospheric drag is always a fixed proportion of the plasma velocity, and thus the "slippage" of the neutral atmosphere always reduces the restframe electric field by a factor $1-K$. This has the same effect as reducing the conductivity by the factor $1-K$, and this behaviour is thus represented by replacing the two uncertain parameters $\Sigma_{P}$ and $K$ by a single "effective conductivity":

$\Sigma_{P}^{*}=(1-K) \Sigma_{P}$

This parameterisation of the upper atmosphere is useful in that it reduces the behaviour of both the thermosphere and ionosphere to a single number that may be used as a free parameter in magnetosphere models (e.g. Saur et al., 2004).

A useful perspective on this model is afforded if we think of $K$ as something similar to a spring constant that represents the degree to which the magnetosphere is able to force the thermosphere into subcorotation. If we double the corotation lag of the magnetosphere, then this model tells us that we also double the corotation lag of the thermosphere; release the drag due to the magnetosphere entirely and the thermosphere "springs" back to rigid corotation under the action of viscosity.

There are several problems with the eddy viscosity model. Firstly, in order to fit the available observations at Jupiter, it was necessary for Huang and Hill (1989) to invoke an enhanced eddy diffusion coefficient to increase the atmospheric viscosity. Since they did not consider the chemical and thermal consequences of enhanced eddy diffusion - we would expect increased eddy activity to raise the altitude of the homopause and increase conductive cooling of the thermosphere - it is not clear that this approach was strictly valid. This criticism is discussed in more detail in Sect. 8. Secondly, they explicitly neglected angular momentum transport by meridional winds, an assumption that was not adequately justified. Thirdly, they ignored any contribution to $K$ from meridional winds coupled to the Hall conductivity.

We will focus on the second point, largely because our existing thermosphere model (Smith et al., 2005; MüllerWodarg et al., 2006) is particularly well suited to the task. The third point could in principle be addressed by our model but, as we shall see in Sect. 3.3, the altitude range of our model does not encompass any regions of significant Hall conductivity.

\section{Model description}

Our model has already been described by Smith et al. (2007). Here we provide as brief as possible a summary of the contents of the model. Here we provide as brief as possible a summary of the contents of the model.

Existing planetary thermosphere models (e.g Achilleos et al., 1998; Bougher et al., 2005) generate complicated results that are difficult to interpret cleanly. For this reason we have attempted to construct a model that is as simplified as possible in order to permit analyses of the fundamental physics of the thermosphere. To this end, we make two key symmetry assumptions: firstly that the entire system is symmetric about the planetary rotation axis (axisymmetry) and secondly that the system is mirror symmetric about the planetary equator. Both of these assumptions are discussed by Smith et al. (2007); we believe that the errors introduced by these symmetry assumptions are more than compensated for by the insights permitted by a simplified model.

The precise rotation period of Saturn remains in doubt. In particular, the SKR period (e.g. Gurnett et al., 2005), which varies over time, differs from periodicities in magnetic field data (e.g. Cowley et al., 2006). Since our primary aim is to investigate the physics of the upper atmosphere, rather than to make any precise numerical predictions, our choice of $\Omega_{S}$ should not have a significant qualitative effect on our results. Therefore, throughout this study we take the preCassini value of $\Omega_{S}=1.638 \times 10^{-4} \mathrm{rad} \mathrm{s}^{-1}$. This is the value 
adopted by the study from which we derive our magnetosphere model (Cowley et al., 2004). It functions as a reference rotation velocity for this model, and so we must adopt it in order to reproduce the correct plasma velocities. It is also the value adopted by previous studies using our thermosphere model (Smith et al., 2005, 2007; Müller-Wodarg et al., 2006). In this case it functions as the rotation period of the lower boundary of the model - adopting the same value ensures that our results are consistent with these previous modelling efforts.

\subsection{Thermosphere model}

The thermosphere model is a global three-dimensional timedependent general circulation model. Full details of the model are given by Müller-Wodarg et al. (2006). The lower boundary is the mesopause, located at a pressure of $0.1 \mu \mathrm{b}$, approximately $800 \mathrm{~km}$ above the $1 \mathrm{bar}$ level. The temperature at this lower boundary is kept fixed at $143 \mathrm{~K}$, the temperature at this altitude in the Moses et al. (2000) model of the neutral atmosphere. Keeping this temperature fixed is intended to represent the efficient radiative cooling by hydrocarbons at these altitudes. We do not include any explicit radiative cooling in our model.

As already mentioned, we assume axisymmetry and mirror symmetry at the equator. We thus reduce the threedimensional model to a two-dimensional model, neglecting all terms involving east-west gradients, and perform calculations only in the Northern Hemisphere. The boundary conditions at the equator are that the meridional gradients of temperature and zonal wind velocity are zero and that the meridional wind velocity is itself zero. Note that the assumption of axisymmetry does not mean that we neglect zonal winds: zonal winds are fully calculated, but are assumed to be identical at all longitudes. The latitudinal resolution of the thermosphere model is $0.2^{\circ}$, corresponding to a distance of $\sim 200 \mathrm{~km}$. Vertically, we employ 55 pressure levels at a resolution of 0.2 pressure scale heights. We adopt an eddy coefficient of $K_{\tau}=10^{4} \mathrm{~m}^{2} \mathrm{~s}^{-1}$ (Atreya, 1982).

\subsection{Magnetosphere model}

We employ the simple axisymmetric magnetosphere model of Cowley et al. (2004). This describes the behaviour of the magnetosphere in terms of the rotation velocities of nested shells of magnetic flux. A flux function $F$ is used to map between the magnetosphere and thermosphere. Since the plasma velocity model is defined in terms of this flux function, we only require the value in the ionosphere for this study. For this purpose we employ the SPV model of Davis and Smith (1990), with two small modifications. Firstly, we neglect the quadrupole term, the principal effect of which is to introduce a small asymmetry between the Northern and Southern Hemispheres; since we assume hemispheric symmetry, it seems reasonable to neglect this term so that our results represent the average behaviour. (Note that our neglect of the quadrupole term is nothing more than a means of imposing hemispheric symmetry; it does not affect the magnetospheric flux function, which we do not need to calculate.) Secondly, our thermosphere model is spherical and thus does not currently take into account effects due to oblateness of the planet, which have a non-negligible effect on the value of the ionospheric flux function. We thus calculate $F_{i}$ as a function of co-latitude $\theta$, using the same model of oblateness described by Cowley and Bunce (2003), and then map these values to identical co-latitudes in our spherical model.

The angular velocity of the magnetosphere $\Omega_{M}$ is then given as a function of $F$ by the following expression:

$$
\begin{aligned}
\Omega_{M}(F) & =\Omega_{A} \\
& +\left(\Omega_{B}-\Omega_{A}\right) T_{A B}(F) \\
& +\left(\Omega_{C}-\Omega_{B}\right) T_{B C}(F) \\
& +\left(\Omega_{D}-\Omega_{C}\right) T_{C D}(F)
\end{aligned}
$$

where the parameters $\Omega_{X}$ represent the nominal rotation velocities of different regions of the magnetosphere which we label for convenience $\mathrm{A}, \mathrm{B}, \mathrm{C}$ and $\mathrm{D}$, and the functions $T_{X Y}(F)$ represent smooth transitions between these regions. The details of these functions are given in the Appendix. Note that we have written this plasma velocity model in a slightly different but mathematically identical form to that originally provided by Cowley et al. (2004).

The physical significance of the various regions of the plasma flow model - which we label A, B, C and D - are discussed in detail by Cowley et al. (2004). Region A is intended to represent field lines open to the solar wind, and thus, for the most part, connected to the planet's magnetotail; region B represents a region of elevated flow in the outer magnetosphere, possibly related to the Dungey cycle return flow and the Vasyliunas cycle; regions C and D represent the mass-loaded middle and inner magnetospheres, respectively, a region which becomes progressively closer to corotation moving towards the planet. The plasma in region D is supposed to be in perfect corotation with the connected neutral atmosphere.

The empirical basis of the model is summarised in Fig. 2. Here the thin, horizontal solid line represents rigid corotation and the thicker solid line represents the model as described by Eq. (14). Data points from the Voyager 1 inbound and outbound passes and the Voyager 2 inbound pass are shown by filled circles, open circles, and crosses (Richardson, 1986, data supplied by the NASA Planetary Data System), respectively. Polar cap plasma velocities determined by Stallard et al. (2004) are shown as horizontal dashed lines on the left hand side of the plot. It is clear that the data is very scattered in some regions, and data from different passes do not agree. For example, the Voyager 2 data points in regions C and D all lie above the plasma velocity curve defined by the model. These points also show clear super-rotation of $\sim 5 \%$ in the region of $22-25^{\circ}$ co-latitude, a feature that is not represented by the Cowley et al. model. 


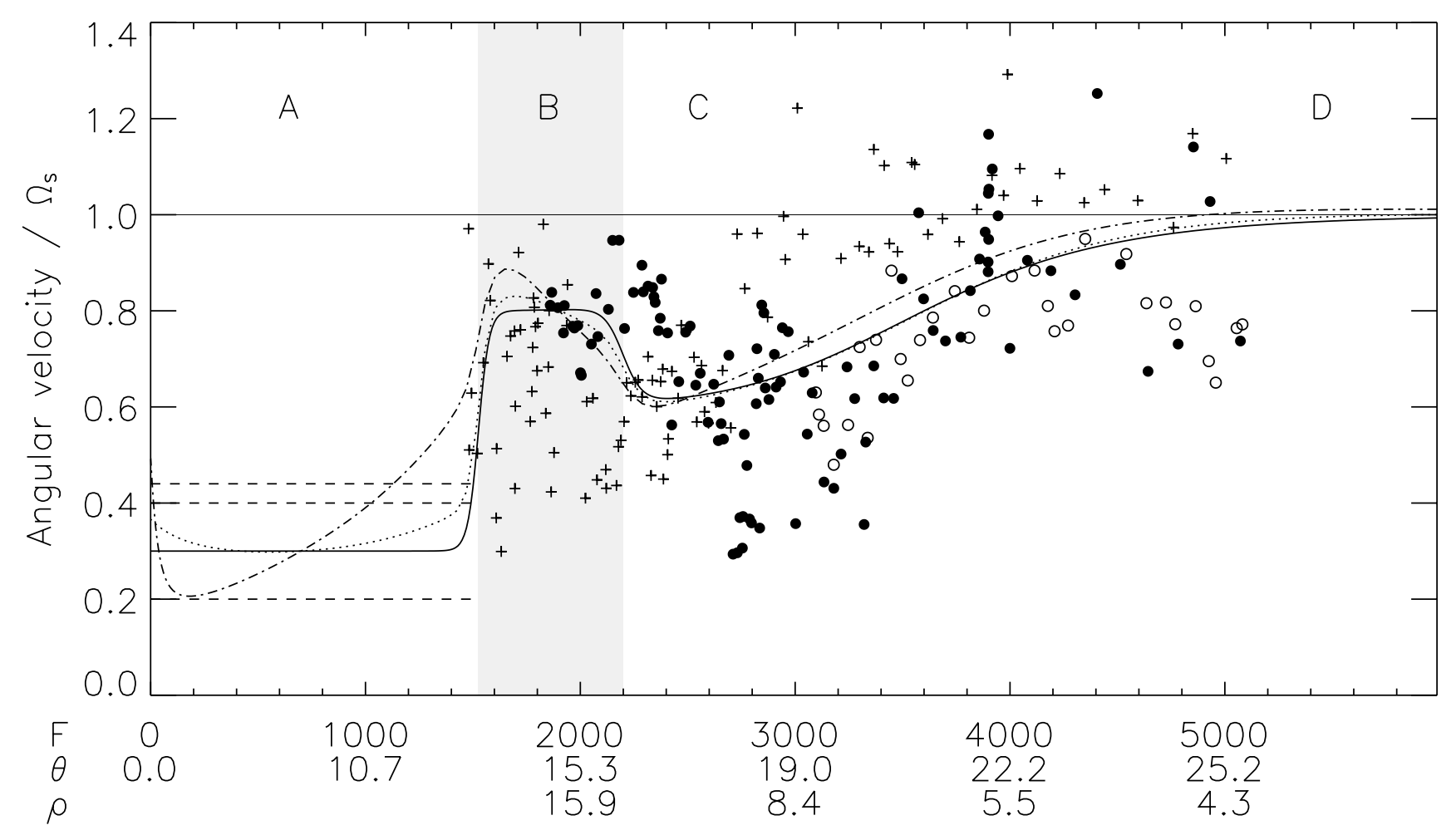

Fig. 2. Empirical basis of the plasma velocity model. Data points from the Voyager 1 inbound and outbound passes and the Voyager 2 inbound pass are shown by filled circles, open circles, and crosses (Richardson, 1986, supplied by the NASA Planetary Data System), respectively. The horizontal dashed lines on the left hand side of the plot show the three polar cap rotation velocities determined by Stallard et al. (2004). The solid line shows the Cowley et al. (2004) plasma velocity model; region B of this model is indicated by the light grey shaded area. The plasma velocities predicted by our modified model (see Sect. 5) for the unscaled and scaled conductivity models are shown by the dotted and dot-dashed lines, respectively. The data is plotted against the magnetic flux function used to map between the magnetosphere and atmosphere. We also show the corresponding co-latitudes $(\theta)$ and $\mathrm{L}$ values $(\rho)$.

Additionally, certain features of the model - for example the sharp shear in the plasma velocity at the boundary between regions $\mathrm{A}$ and $\mathrm{B}$ - are not strictly present in the data but are consequences of the physical model of Cowley et al. (2004) that associates the main auroral oval with the openclosed field-line boundary. Other models consistent with the data are thus possible. Due to this ambiguity, our approach is to take the Cowley et al. plasma flow model "as is" and use it as a context for investigating the dynamical coupling of the thermosphere and magnetosphere, while recognising that other models may be equally compatible with the data.

Note that we initially apply the model as a fixed boundary condition to our thermosphere model. However, this leads to various inconsistencies, discussed in Sect. 4, which we remedy using the modified model described in Sect. 5.

\subsection{Ionospheric conductivity}

To couple together the thermosphere and magnetosphere models using the expressions laid out in Sect. 2 we require a model of the Pedersen and Hall conductivities; to calculate these we require a model of the ion and electron densities in the ionosphere. There are no reliable empirical or theoretical models of Saturn's global ionosphere: both the available data and our understanding of the physical and chemical processes affecting the ionosphere are at present inadequate to provide such a model. It is thus likely that any ionosphere model we adopt will be to some extent incomplete or inaccurate.

For this reason we think that detailed ionosphere modelling is a distraction from our main objective which is to understand the coupling between the dynamics of the thermosphere and magnetosphere. We therefore use the output from one particular ionosphere model, calculate the conductivity, and then fix it. Thus we neglect feedback to the conductivity from any changes in the thermal and dynamical structure of the thermosphere. Not only does this approach considerably improve the runtime and stability of our numerical model as compared to fully coupled thermosphere-ionosphere models, it also gives us confidence that the results of the model represent processes of purely thermospheric origin. 


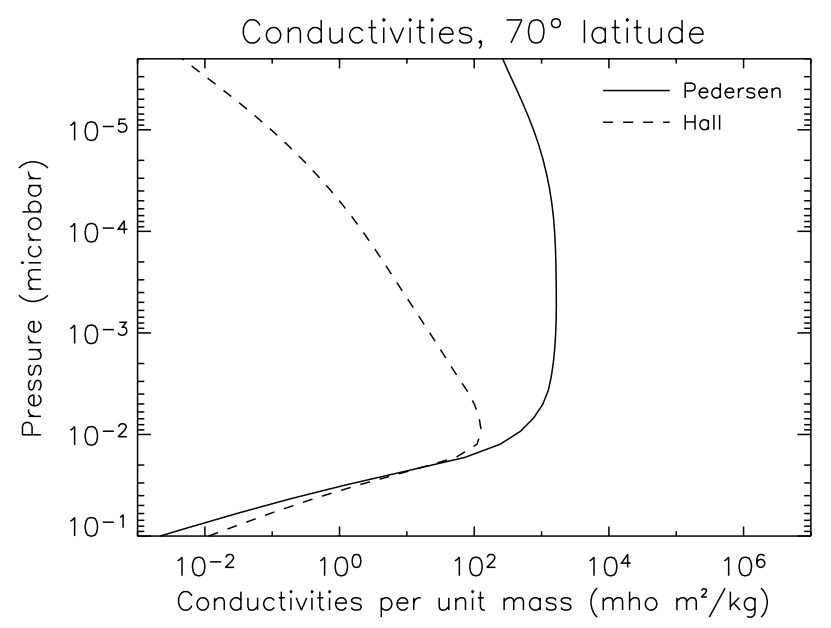

Fig. 3. Conductivity profiles at $70^{\circ}$ latitude. Solid lines: Pedersen conductivity per unit mass. Dashed lines: Hall conductivity per unit mass.

The question then arises as to how one should fix the conductivity in a thermosphere model that exhibits variable thermal structure. Our solution is to calculate $\sigma_{P}$ and $\sigma_{H}$ using a particular set of ion and neutral densities, and then fix, as a function of pressure, the quantities $s_{P}=\sigma_{P} / \rho$ and $s_{H}=\sigma_{H} / \rho$, where $\rho$ is the neutral mass density. The advantage of fixing these quantities is that the height-integrated conductivities are then also fixed, since, for example:

$\Sigma_{P}=\int_{z 0}^{z 1} \sigma_{P} d z=\int_{z 0}^{z 1} s_{P} \rho d z=\int_{p 1}^{p 0} s_{P} g d p$

where the last step follows from hydrostatic equilibrium $d p / d z=-\rho g$, where $g$ is the acceleration due to gravity. If $g$ is constant with height (a good approximation since the vertical extent of the thermosphere is small compared to the radius of the planet) and $s_{P}$ is specified as a fixed function of pressure, then the RHS must be fixed, and therefore $\Sigma_{P}$ is also fixed. An identical argument applies for $\Sigma_{H}$. Thus fixing the quantities $s_{P}$ and $s_{H}$ - which we may usefully refer to as conductivities "per unit mass" - allows us to control the height-integrated value of the conductivity regardless of any changes in the thermal structure of the upper atmosphere.

To calculate the conductivity we use $\mathrm{H}^{+}$and $\mathrm{H}_{3}^{+}$densities from the ionosphere model of Moore et al. (2004). The version of the model used here includes ionisation due to solar insolation only. For this study the model has been run in an equinox configuration, at solar maximum, using the neutral atmosphere of Moses et al. (2000) as a fixed global background. The resultant ion densities are then used to calculate the conductivities $\sigma_{P}$ and $\sigma_{H}$, using the Moses et al. (2000) neutral atmosphere. Our expressions for $\sigma_{P}$ and $\sigma_{H}$ are given in the Appendix.

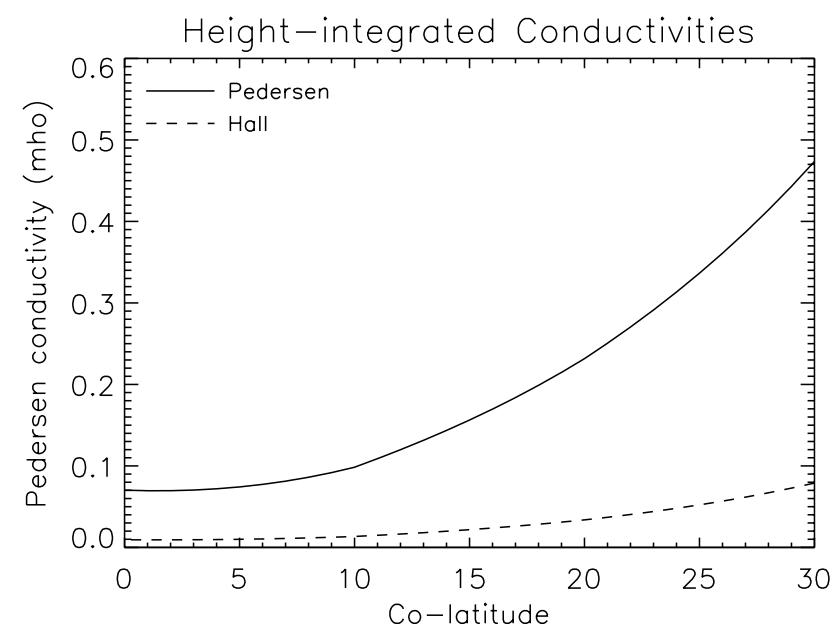

Fig. 4. Height-integrated conductivities. Solid lines: Pedersen conductivity. Dashed lines: Hall conductivity.

The resultant conductivity profiles for $70^{\circ}$ latitude are plotted in Fig. 3 and the distribution of the height-integrated conductivities with latitude in Fig. 4. It is clear that the Pedersen conductivity is more important than the Hall for almost the entire vertical extent of our model, and is more important in a height-integrated sense throughout the polar regions. This indicates that the coupling between Hall conductivity and meridional winds described in Sect. 2 is unlikely to be important in this study, unless the meridional winds are much greater than the zonal winds. If we were to extend our model downwards into the mesosphere, though, it is possible that this situation would change.

The cut-off in both conductivity profiles at approximately $0.01 \mu$ bar corresponds to the homopause. Below this level hydrocarbon densities increase quickly with decreasing altitude. These hydrocarbons undergo rapid charge exchange with $\mathrm{H}^{+}$and $\mathrm{H}_{3}^{+}$; the resultant hydrocarbon ions then recombine relatively quickly. The result is a depletion in the ionospheric densities and conductivities at this level.

The values of $\Sigma_{P}$ predicted in the polar regions lie in the range $0.1-0.5 \mathrm{mho}$. These values are an order of magnitude below the value of $\Sigma_{P}^{*}=1 \mathrm{mho}$ adopted by Cowley et al. (2004). We will discuss this difference in Sect. 7; we do not think it helpful at this stage to "fudge" our ionosphere model to remove the discrepancy.

\subsection{Coupled model}

The coupled model consists of the thermosphere model driven by the values of Joule heating and ion drag implied by the magnetosphere and ionosphere models. These values are updated at each timestep. We find that the model reaches near dynamical equilibrium much more quickly than near thermal equilibrium. As shown by Smith et al. (2005), the time for 
the latter is approximately 400 planetary rotations. We adopt this runtime for this study.

\section{Initial model results}

We now describe the output of the model detailed above. First we describe the basic thermospheric response that has been discussed already by Smith et al. (2007). We then compare the rotational structures of the thermosphere and magnetosphere and suggest that we must modify the magnetosphere model if our results are to make sense.

\subsection{Thermospheric response}

Figure 5 summarises the response of the polar thermosphere to forcing by the magnetospheric drag. The essential features of this response have been discussed by Smith et al. (2007). The magnetospheric drag drives winds that are primarily westwards and polewards. The polewards flow is particularly important, in that it concentrates the energy injected from the magnetosphere into a "hotspot" at the pole and acts to cool mid-latitudes. Smith et al. (2007) showed that increasing the ionospheric conductivity only intensified this behaviour, and thus concluded that the energy injected by the magnetosphere could not be responsible for heating midlatitudes.

For this study we are particularly interested in the interaction between the neutral winds and the conductivity distribution. The figure clearly shows that most of the conductivity is concentrated below $1200 \mathrm{~km}$ across the whole of the polar thermosphere. Looking at the meridional winds, it is clear that below $1200 \mathrm{~km}$ they are almost universally polewards. These polewards meridional winds may be responsible for transporting angular momentum from mid-latitudes into the polar regions; we will return to this suggestion below.

\subsection{Rotational structure}

The output of our initial model run is summarised in Fig. 6a, which shows the rotational velocity of the magnetosphere $\Omega_{M}$ and the rotational velocity of the thermosphere $\Omega_{T}$. We also show the anti-corotational torque on the thermosphere (Fig. 6b), the true and effective height-integrated Pedersen conductivities (Fig. 6c) and $K$. Each plot also shows, with a dotted line, values implied by a constant value of $K=0.4$.

Figure 6 clearly shows that our model predicts structures that are not present in the constant $K$ model. Looking first at the rotation velocities (Fig. 6a) we can see that the neutral rotation velocity predicted by the model is very different to that for a constant value of $K$. If $K$ is a constant, shown with the dotted line, the neutral rotation velocities in each flow region are constant and follow the sharp gradients in the plasma velocity exactly, by definition. In contrast, the velocities predicted by the model are not constant across each flow region, but vary slowly and smoothly with latitude. At boundaries between plasma flow regions the neutral velocity does not change sharply with the plasma velocity, but again varies smoothly across the boundary. At first glance, we can characterise this behaviour as a latitudinal "smearing" of the constant- $K$ curve.

The "smeared" form of the neutral rotation velocity profile produces two unexpected and interesting features. Firstly, the latitudinal profile of $K$ is "wiggly", as shown in Fig. 6d. The variations in $K$ are particularly pronounced around region $\mathrm{B}$, where the plasma velocity varies most rapidly with latitude. The "average" value of $K$ is $\sim 0.4$, but it exhibits values throughout the range $0.2-0.6$ in this region. Secondly, at $25^{\circ}$ co-latitude $K$ becomes negative. This is because the neutrals super-rotate in this region, such that the "slippage" of the neutral atmosphere represented by $K$ is negative. We now analyse these two unexpected features of the rotational flow in greater detail.

\subsubsection{Latitudinal "smearing"}

Before continuing we must eliminate the possibility that the latitudinal "smearing" of the neutral velocities relative to the plasma velocities is an artefact of the model rather than a real physical effect. There are two non-physical effects in the model that may generate latitudinal smoothing of the zonal winds. Firstly, the latitude grid step size provides a limit to the scale size of processes that can be accurately represented by the model. Attempting to force the model to produce gradients or shears with scale sizes smaller than the latitude grid step will produce a "smoothed" response.

Secondly, the model incorporates a numerical smootherdesmoother algorithm to filter out numerical noise at the spatial frequency of the latitude grid step size (Fuller-Rowell et al., 1996, and references therein). If there is a sharp gradient in the zonal winds that occurs on a spatial scale close to the grid step, the gradient will contain spatial frequencies close to the grid step size, and these will be removed by the smoothing algorithm, effectively smoothing out the gradient.

Both of these processes are, ultimately, consequences of an over-large grid step size. Since the transitions in the plasma velocity at the inner and outer edges of region B occur over a spatial scale of $\sim 1^{\circ}$, and our standard grid resolution is $0.2^{\circ}$, our resolution is close to the level at which it may fail to resolve the velocity shears correctly. To check that non-physical processes are not responsible for the latitudinal smearing, the model has therefore been run for a further 10 rotations, at a latitude grid step of $0.025^{\circ}$, through the latitude range $70-80^{\circ}$, using the previous, lower resolution run as a startup atmosphere and boundary condition. We find that 10 rotations is enough for this model to make the (very small) adjustment required for it to reach near steady-state.

The results of this run are shown in Fig. 7. Figure 7a shows the quantities $\Omega_{M}$ (dashed line) and $\Omega_{T}$ (solid line). The value of $\Omega_{T}$ calculated with the standard model is shown with the dot-dash line. This figure shows that using the 


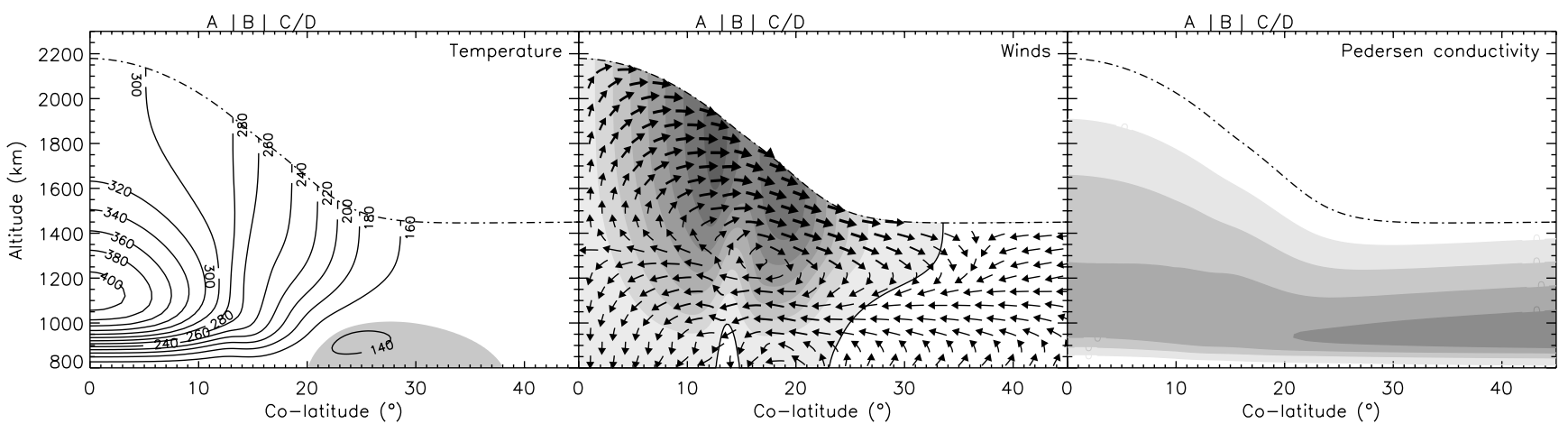

Fig. 5. Thermospheric response of our initial model. Left: temperature structure. The shaded region is cooler than the lower boundary temperature of $143 \mathrm{~K}$. Centre: winds. Arrows show the combined meridional and vertical circulation. Shading shows the magnitude of the zonal wind: regions shaded grey represent eastwards (subcorotational) winds; regions not shaded represent westwards (supercorotational) winds. The solid contour represents zero zonal wind (perfect corotation). Right: Pedersen conductivity distribution. The darkest region represents conductivities greater than $10^{-6} \mathrm{mho} / \mathrm{m}$; the next darkest region conductivities greater than $10^{-7} \mathrm{mho} / \mathrm{m}$ and so on until the unshaded region represents conductivities less than $10^{-9} \mathrm{mho} / \mathrm{m}$.

very high resolution model does not eliminate the latitudinal smearing effect. The $\Omega_{T}$ curve is slightly less smeared in the high-resolution case, exhibiting a slightly sharper peak at $13.5^{\circ}$ colatitude. We may ascribe this to a slight reduction in the importance of the non-physical smoothing effects. However, the profile is still substantially smeared, and it is clear that these non-physical effects are not the dominant contribution to the smearing effect.

In Fig. $7 \mathrm{~b}$ we show the height-integrated balance of the various torques acting on the thermosphere in the highresolution run. We group the torques into ion drag (solid line), Coriolis and curvature (dotted line), advection (short dashed line) and viscosity (long dashed line). In addition to plotting these four groups of physically meaningful terms we also plot their residual, i.e. the imbalance of the torques calculated by the model (dot-dash line). This residual represents deviations from equilibrium caused either by the model not having run to steady state, or by the non-physical smoothing terms.

It is clear first of all that the residual is relatively insignificant, lying close to zero throughout the range shown. This immediately indicates, as suggested by Fig. 7a, that the smearing is a real physical process, not a modelling artefact; it also supports our assertion that the model is very nearly in steady state. Secondly, we can see that the viscosity term is also relatively insignificant, again lying close to the zero line.

The significant terms are therefore ion drag, Coriolis and advection. Before we describe the balance between these three components the reader is reminded that the prevailing wind at low altitudes, where most of the inertia and conductivity are located, is towards the pole (Fig. 5). Thus advection tends to transport quantities from right to left in the figures. Starting from the right hand side ( $18^{\circ}$ colatitude) it is clear that Coriolis is the most important term balancing ion drag.
As the wind moves into region B (the shaded area) it encounters a reduced ion drag, due to the rise in the plasma velocity. This reduction in ion drag makes advection a more important sub-corotational term. This is because the gas arriving at the edge of region $\mathrm{B}$ is sub-corotating to a greater extent than the plasma to which it is about to become connected. As it moves into and across region B it accelerates gradually towards corotation, driven by the Coriolis force. This slow spin-up as the gas moves into and then through region B is the process that generates the smearing at this first boundary.

At the polewards boundary of region B the same process occurs, but in the opposite sense. Gas that has moved back towards corotation moving through region B suddenly encounters much more strongly sub-corotating plasma in the polar cap (region A). At this juncture advection acts to support the flow, as the inertia of the polewards flowing gas prevents the neutral velocity dropping as quickly as the plasma velocity. Thus the neutral velocity falls off slowly towards the pole, as the neutrals are gradually accelerated into subcorotation by the plasma. This smoothing of the neutral profile by the combined influence of advection and Coriolis leads to a profile that is not only smoothed but also slightly shifted towards the pole.

The difference between the neutral angular velocity profiles calculated with the low and high resolution models is consistent with this description of the origin of the smearing. If the smearing is caused by meridional advection, then a parcel of neutral gas should only respond to a shear in the plasma velocity when it reaches that shear. Thus at the A-B boundary, the velocity of the neutral gas moving towards the pole should not begin to drop before that of the plasma, since it has no mechanism with which to detect the change in the plasma velocity any earlier. The high resolution model does indeed exhibit this behaviour, whereas the neutral velocity in 
(a)
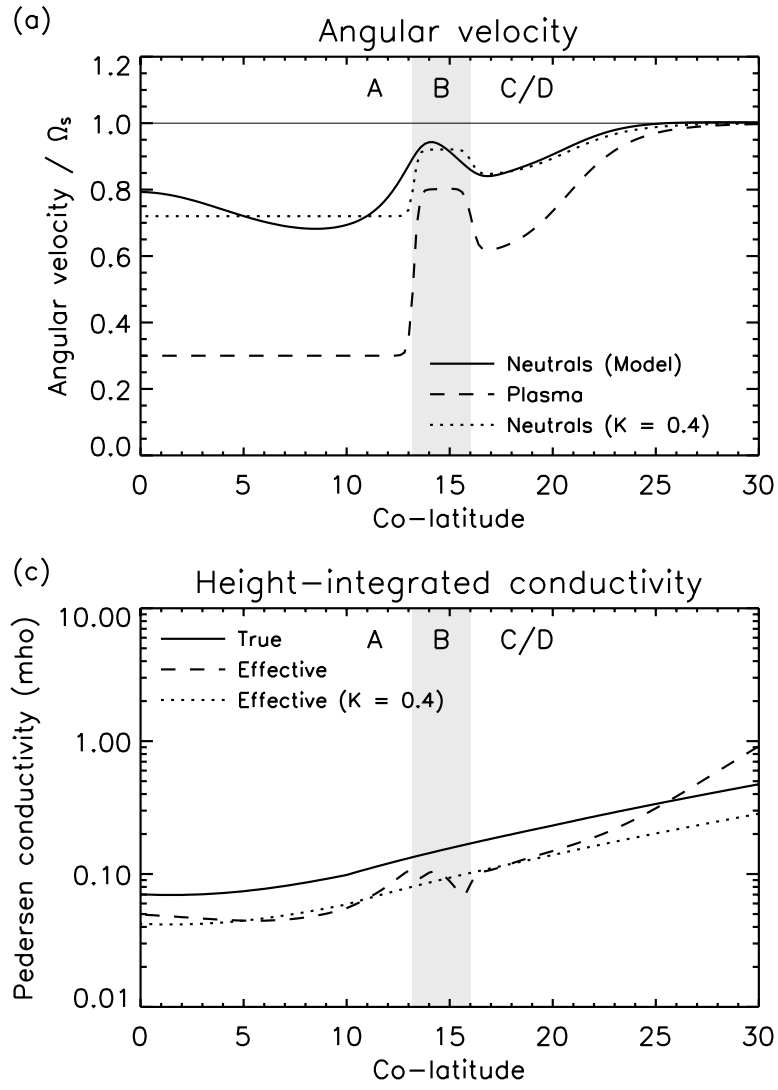

(b)

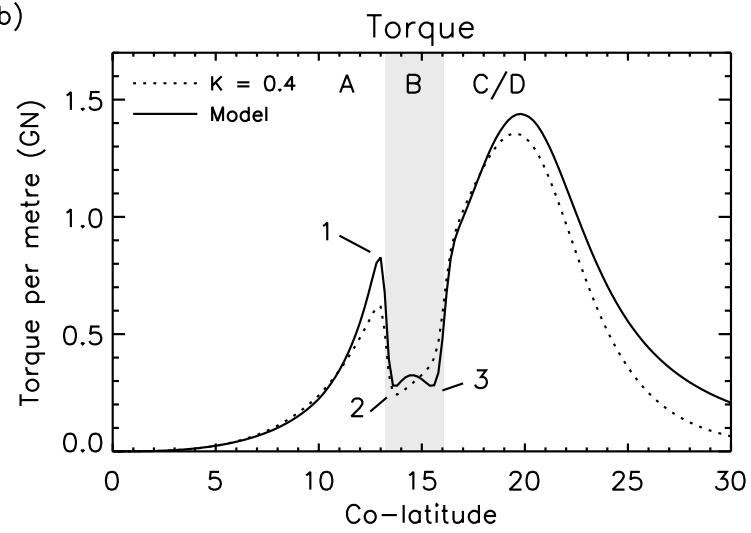

(d)

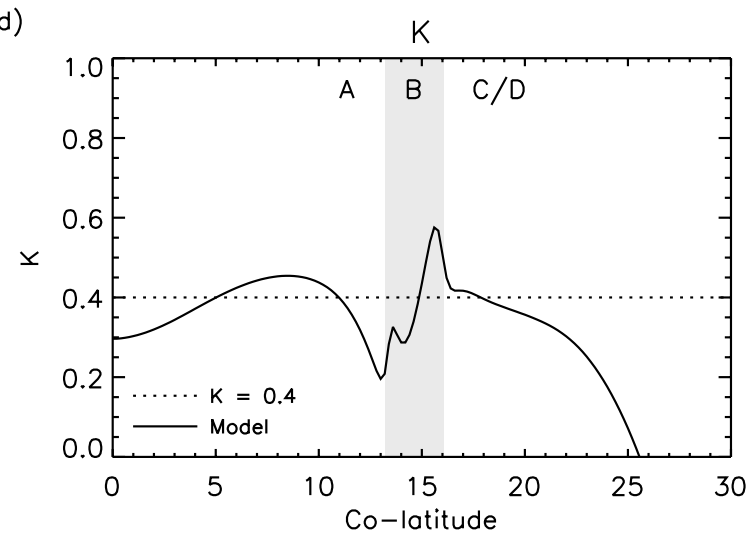

Fig. 6. Parameters for our initial magnetosphere model ( $\Omega$-model). In each plot the grey shaded area represents region B of the magnetosphere model. (a) Angular velocities. Solid line: height-integrated thermospheric angular velocity $\Omega_{T}$, calculated using the thermosphere model. Dashed line: plasma angular velocity $\Omega_{M}$. Dotted line: height-integrated thermospheric angular velocity $\Omega_{T}$ inferred from $\Omega_{M}$ if $K=0.4$. (b) Anti-corotational (corotational) torque exerted by the magnetosphere (thermosphere) on the thermosphere (magnetosphere), as a function of latitude in the thermosphere. The torque is expressed in units of torque per metre of latitude, integrated in azimuth. Solid line: torque calculated using thermosphere model; dotted line: torque inferred if $K=0.6$. (c) Height-integrated Pedersen conductivity, $\Sigma_{P}$ (solid line). The effective height-integrated Pedersen conductivity $\left(\Sigma_{P}^{*}\right)$ is shown by the dashed line. Dotted line: $\Sigma_{P}^{*}$ is $K=0.4$. (d) The thermospheric rotation parameter $K$, as a function of latitude. Solid line: $K$ calculated from thermospheric model. Dotted line: $K=0.4$.

the low resolution model clearly begins to drop before the plasma velocity. This is presumably a result of the numerical smoothing, which, by acting diffusively, allows the gas to be affected by the change in the plasma velocity before it reaches the boundary.

Looking now at the viscosity curve, we can see that not only is it relatively insignificant - never contributing more than about $20 \%$ of the force balance at any latitude - but that it does not systematically oppose ion drag. In the range $13-15^{\circ}$ co-latitude the net effect of viscosity is to act in the same direction as ion drag. This is related to the small region of super-corotation at these co-latitudes close to the lower boundary of the model (Fig. 5, centre panel). Thus, not only is viscosity less important than previously supposed, its influence on the structure of the thermosphere is also much more complicated.
The preceding analysis has two important consequences. Firstly, we have shown that the viscosity is almost entirely insignificant as a means of supplying angular momentum to the high-latitude thermosphere. The angular momentum extracted by ion drag is replaced by advection of angular momentum from lower latitudes. Thus the eddy viscosity model of Huang and Hill (1989) and Pontius (1995) is shown to be false in the circumstances represented by our model. It is, of course, possible that the eddy diffusion coefficient is significantly enhanced in the polar regions such that angular momentum transport due to eddy viscosity is comparable with that due to advection. However, there is no direct evidence for such an enhancement, nor is there any reason to invoke such an enhancement except as a post hoc defence of the eddy viscosity model.

This new model for angular momentum transfer is sketched in Fig. 8. The top panel represents the old eddy 

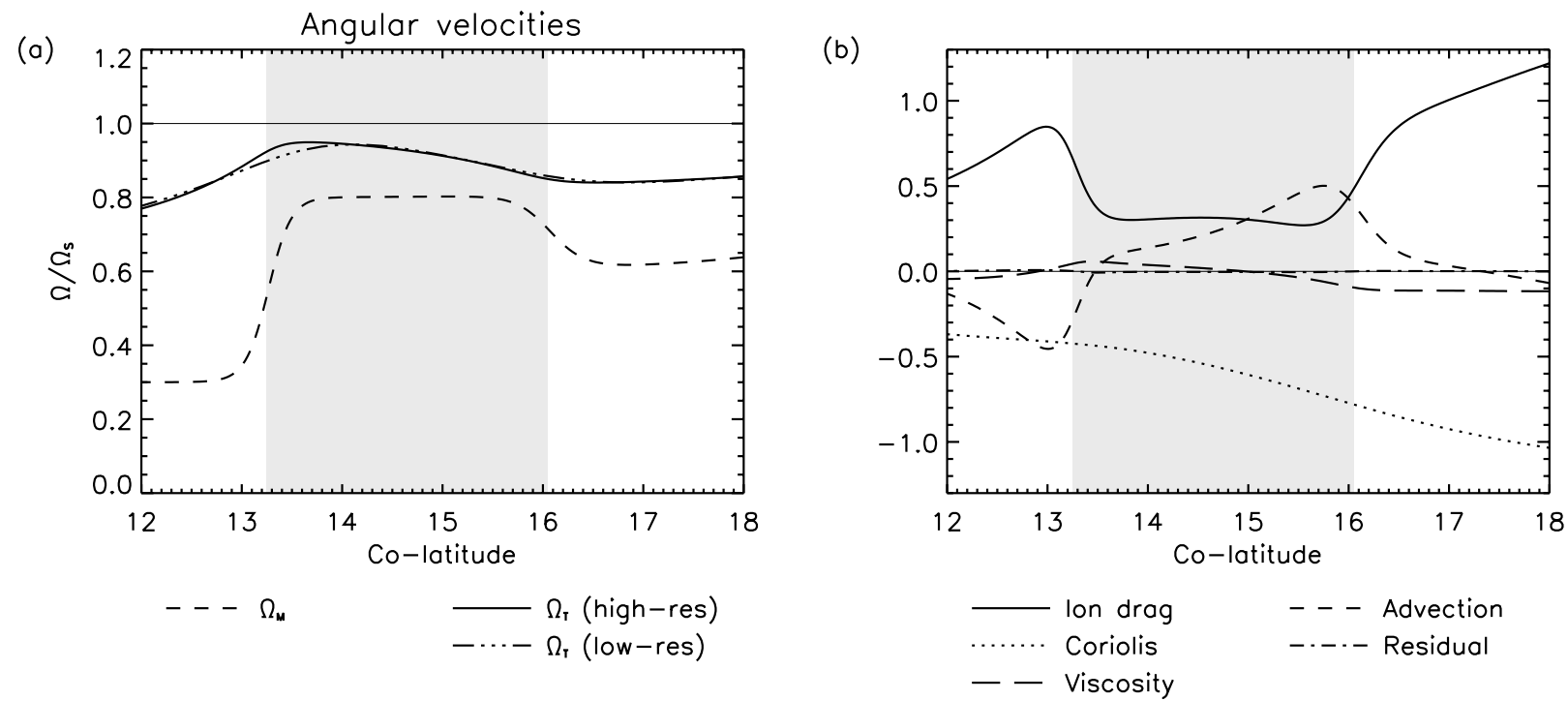

Fig. 7. Parameters of nested grid model. The shaded region on each plot represents region B of the plasma flow model. (a) Angular velocities. Neutral velocities are shown by the dash-triple-dot and solid lines for the low- and high-resolution models, respectively. The dashed line shows the fixed model of $\Omega_{M}$. (b) Height-integrated, normalised anti-corotational torques acting on the neutral atmosphere, in the same units as Fig. 6: ion drag (solid line), Coriolis and curvature (dotted line), advection (short dashed line), viscosity (long dashed line) and residual (dot-dashed line).

viscosity model in which angular momentum was supplied vertically at each latitude by the action of viscosity, independent of the behaviour of the atmosphere at adjacent latitudes. The lower panel shows our new model, in which angular momentum is transported into the thermosphere by upwelling at mid-latitudes and transported polewards by meridional winds. The flow then returns to the lower atmosphere through downwelling at the pole. Note that due to the effect of Coriolis in spinning up the gas as it flows towards the pole, this new model does not require any viscous transfer of angular momentum into the thermosphere, even at low latitudes.

The second consequence of the above analysis is that the latitudinal smearing of the neutral winds is a real physical effect. The significance of this becomes apparent on consideration of the torque exerted on the thermosphere by the magnetosphere, as shown in Fig. 6b. This must of course be equal to the anti-corotational torque exerted on the magnetosphere by the thermosphere. The troughs and peaks of $K$ are replicated in the latitudinal profile of torque. In particular, the torque drops to small values close to the inner edges of region B (labels 2 and 3), and just outside the poleward edge, the torque is strongly peaked (label 1). These structures are not observed if $K$ is constant, as shown by the dotted line.

These peaks and troughs are important because the model we have constructed is intended to be a simple representation of the mean, steady state configuration of the coupled thermosphere-magnetosphere system. If the thermosphere exerts the complex corotational torque shown in Fig. 6b upon the magnetosphere, an equally complex anti-corotational torque must be exerted on the magnetosphere by some other process or set of processes to ensure steady-state. This anticorotational torque must either be an inertial effect - i.e. a result of mass-loading or radial motions of plasma - or associated with solar-wind coupling.

To support the model of Cowley et al. (2004) thus requires us to postulate the existence of such a torque - of precisely the form shown - in order to maintain the regions of constant rotational velocity. It is possible in principle that such a torque might exist. Region $\mathrm{B}$ maps to the range $\sim 15-$ $20 R_{S}$ in the magnetosphere. The outer edge of this range corresponds to the orbit of Titan, which may be responsible for some mass loading of the magnetosphere. Region B is also by definition adjacent to the magnetopause, such that complex torques due to solar wind coupling may be present. However, while it is possible in principle for the correct torque to exist, it seems extremely unlikely that it does. The data on which the Cowley et al. (2004) model is based is rather scattered, and the inclusion of approximately constant regions of flow was made for reasons of simplicity. If there existed finely tuned torques in the magnetosphere that produced such regions of constant flow velocity, it would be an extraordinary coincidence.

The important insight is that the "wiggles" are not a real physical effect at all, but the result of inflexibly coupling the thermosphere and magnetosphere models. The torques exerted on the magnetosphere by the thermosphere are acting 

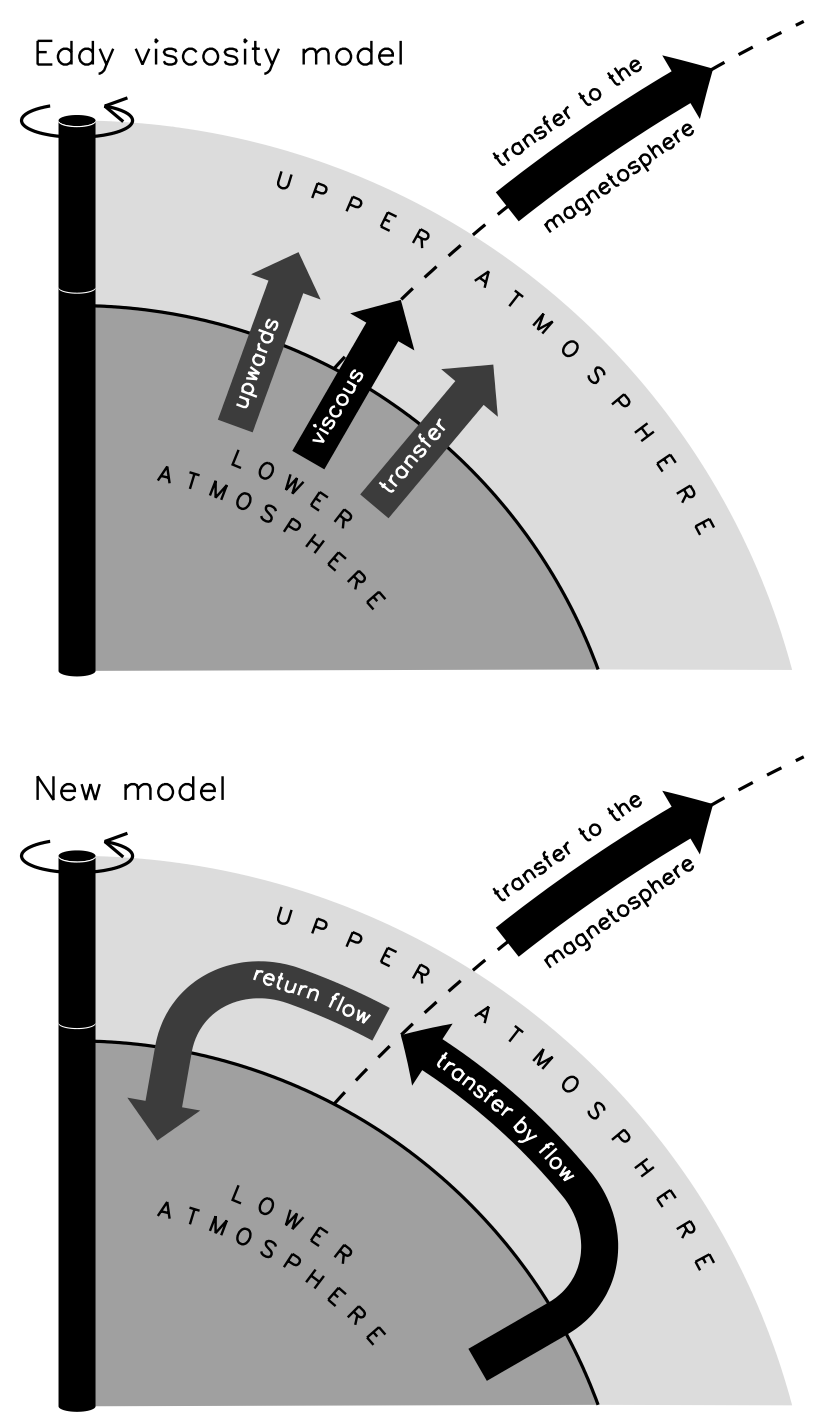

Fig. 8. Models of angular momentum transfer to the upper atmosphere. Top: Eddy viscosity model. In this model angular momentum is transferred vertically from the lower to the upper atmosphere by the action of eddy viscosity. This angular momentum is then electromagnetically transferred to the magnetosphere. Bottom: New model. In this model angular momentum is transferred from the lower to the upper atmosphere by bulk upwards flow of gas at mid-latitudes. This angular momentum is then transferred polewards by meridional winds. Some angular momentum is extracted and transferred electromagnetically to the magnetosphere. The angular momentum depleted flow then returns to the lower atmosphere.

to remove the regions of constant flow, but this is prevented by the fixed nature of the plasma flow model. To improve the realism of our modelling we must modify the plasma flow model so that it is able to respond more elastically to the thermospheric structure. Our adopted modifications are described in Sect. 5.

\subsubsection{Super-rotation}

The second unexpected feature of the flow is the superrotation observed equatorwards of $\sim 25^{\circ}$ co-latitude. This super-rotation is directly linked to the polewards flow of gas shown in Fig. 5. As shown above, this flow is subject to a Coriolis force that tends to spin it up towards corotation, but this force is counteracted by the large subcorotational ion drag in this region.

However, equatorwards of $\sim 25^{\circ}$ co-latitude this drag falls quite rapidly to zero. Thus the zonal wind speed also falls rapidly, and therefore the polewards meridional winds also fall. There is thus a positive divergence in the meridional winds at this co-latitude which leads to upwelling of gas; this upwelling gas cools adiabatically, generating the cool region at $\sim 25^{\circ}$ co-latitude visible in Fig. 5. Equatorwards of this cool region there is now a polewards pressure gradient that drives polewards flow. This flow is also subject to a corotational Coriolis force, but in this region the ion drag is insignificant, and there is thus no force to prevent the gas being driven into super-rotation by the Coriolis force. When the flow reaches $\sim 25^{\circ}$ co-latitude the ion drag begins to become important and the gas begins to sub-corotate.

The existence of this super-rotating region highlights another problem with our fixed magnetosphere model. The model is designed such that in region D the plasma almost corotates with the neutrals. However, "corotation" is implicitly defined as the rotation velocity of the lower atmosphere i.e. the rotation velocity of the lower boundary of our model. We find that corotation with the neutrals in this region actually corresponds to a small degree of super-rotation of the magnetosphere. Such super-rotation is observed in the data (Richardson, 1986; Sittler et al., 2006). This effect is not currently incorporated in the magnetosphere model.

\section{Modified magnetosphere model}

In the preceding section we have identified two flaws with the fixed magnetosphere model described in Sect. 3. Firstly, the latitudinally smooth response of the neutral rotation velocity to the simple plasma velocity profile implies complex torques in the magnetosphere that do not satisfy simplicity considerations. Secondly, it requires that the inner magnetosphere corotate with the planetary angular velocity, not with the true angular velocity of the connected neutral atmosphere. This means that the model is unable to represent super-rotation of the inner magnetosphere driven by the neutral atmosphere. We are thus motivated to modify the plasma velocity model in such a way that the complicated behaviour of the neutral winds is taken into account.

Our modified model follows from two observations. Firstly, the torque required to maintain a rotation velocity $\Omega_{M}$ should be roughly proportional to that rotation velocity; secondly, the torque exerted by the thermosphere that might 
maintain this rotation velocity is proportional to $\Omega_{T}-\Omega_{M}$. We can thus equate these two quantities, with some suitable constant of proportionality $\alpha$ :

$\Omega_{M}=\alpha\left(\Omega_{T}-\Omega_{M}\right)$

We expect this quantity $\alpha$ to depend on the physical conditions in the magnetosphere and ionosphere that correspond to each shell of flux tubes. A large value of $\alpha$ means that a relatively small velocity difference $\Omega_{T}-\Omega_{M}$ is required to enforce rotation at the velocity $\Omega_{M}$ : this might correspond, for example, either to a large ionospheric conductivity or to very low mass-loading of the magnetosphere.

We may re-express this relation:

$\Omega_{M}=\Omega_{T} \frac{\alpha}{1+\alpha}$

Thus, if $\alpha \rightarrow \infty$, then $\Omega_{M} \rightarrow \Omega_{T}$ and the magnetosphere corotates with the thermosphere. If $\alpha \rightarrow 0$, then $\Omega_{M} \rightarrow 0$ and the magnetosphere acquires a negligible rotation velocity.

It is convenient to write the ratio between the magnetospheric and thermospheric velocities as a parameter which we call $\chi$ :

$\chi=\frac{\Omega_{M}}{\Omega_{T}}$

This parameter is conceptually somewhat similar to $K$. The traditional description of the thermospheric behaviour using a constant value of $K$ packages a variety of complex thermospheric processes into a single parameter which represents "slippage" from the thermosphere's natural state of perfect corotation. The new parameter $\chi$ likewise parameterises the complex behaviour of the magnetosphere, representing "slippage" of the magnetosphere from its natural state of almost zero corotation.

To improve the plasma flow model, we now make the assumption that this quantity $\chi$ is approximately constant across each of the flow regions defined by Cowley et al. (2004). This is, of course, a simplification. However, given the lack of better physical models for the plasma flow, especially in region $\mathrm{B}$, such a simplification seems justified and, we think, still represents an improvement over the fixed plasma flow model.

We then simply replace Eq. (14) with an equivalent model for $\chi$ :

$$
\begin{aligned}
\chi(F) & =\chi_{A} \\
& +\left(\chi_{B}-\chi_{A}\right) T_{A B}(F) \\
& +\left(\chi_{C}-\chi_{B}\right) T_{B C}(F) \\
& +\left(\chi_{D}-\chi_{C}\right) T_{C D}(F)
\end{aligned}
$$

where the functions $T_{X Y}$ are unchanged. We may then determine $\Omega_{M}$ at any point by combining Eqs. (18) and (19). Henceforth we will refer to this modified model as the " $\chi$ model" and our initial model as the " $\Omega$-model".

It is clear that we also require values for the parameters $\chi_{A-D}$ that define the actual flow velocities generated by the model. It is important that we continue to be guided by the empirical considerations that determine the flow velocities in the Cowley et al. (2004) model. Thus we define three "anchor points" $F_{A-C}$ in each of the flow regions A-C. We demand that the flow velocity at each of these points is identical to that in the original model. This is enough to specify the parameters $\chi_{A-C}$. We set $F_{A}=700 \mathrm{nTR}_{\mathrm{S}}^{2}$ and $F_{B}=1900 \mathrm{nTR}_{\mathrm{S}}^{2}$ such that the anchor points lie approximately in the "middle" of each region in terms of magnetic flux. We set $F_{C}=2400 \mathrm{nTR}_{\mathrm{S}}^{2}$ such that the minimum rotational velocity in this region lies at approximately the same latitude as in the original model. Finally we fix $\chi_{D}=1$ such that the inner magnetosphere perfectly corotates with respect to the thermosphere. We recalculate the flow velocities implied by this model after each timestep of the thermosphere model, such that the magnetospheric rotation is continually updated with reference to any changes in the thermospheric flow.

\section{Modified model results}

\subsection{Thermospheric response}

The thermospheric response of the modified model is shown in Fig. 9. This is the model output shown in Fig. 1 of Smith et al. (2007). The structures here are almost indistinguishable from those of Fig. 5 . Replacing the $\Omega$-model with the $\chi$-model has increased the temperature of the polar hotspot by $\sim 10 \mathrm{~K}$, a change of only $\sim 2.5 \%$ of the absolute value of $\sim 400 \mathrm{~K}$. Thus the modification is shown to have only a small influence on the thermal structure, justifying the assertion of Smith et al. (2007) that the use of the modified model had an insignificant effect on their results.

\subsection{Rotational structure}

Figure 10 shows the resultant structure of both $\Omega_{M}$ and $\Omega_{T}$ in the same format as Fig. 6; the "anchor points" are shown by the diamonds in panel (a). It can be seen that the thermospheric response is very similar. The sharp gradients in the magnetosphere model have been smoothed in response to the thermospheric structure, but the basic shape of $\Omega_{M}$ remains similar to the previous fixed model. This curve is also plotted in Fig. 2 (dotted line), to allow comparison with the data on which the original $\Omega$-model was based. It is clear that the predictions of the $\chi$-model remain reasonably consistent with all of the available data.

\subsubsection{Latitudinal "smearing"}

Our first motivation for introducing the $\chi$-model was to remove the "wiggly" profile of the torque exerted on the magnetosphere by the thermosphere. This problem has been corrected, since Fig. 10b is now a smooth function everywhere, consistent with our desire to represent approximately 


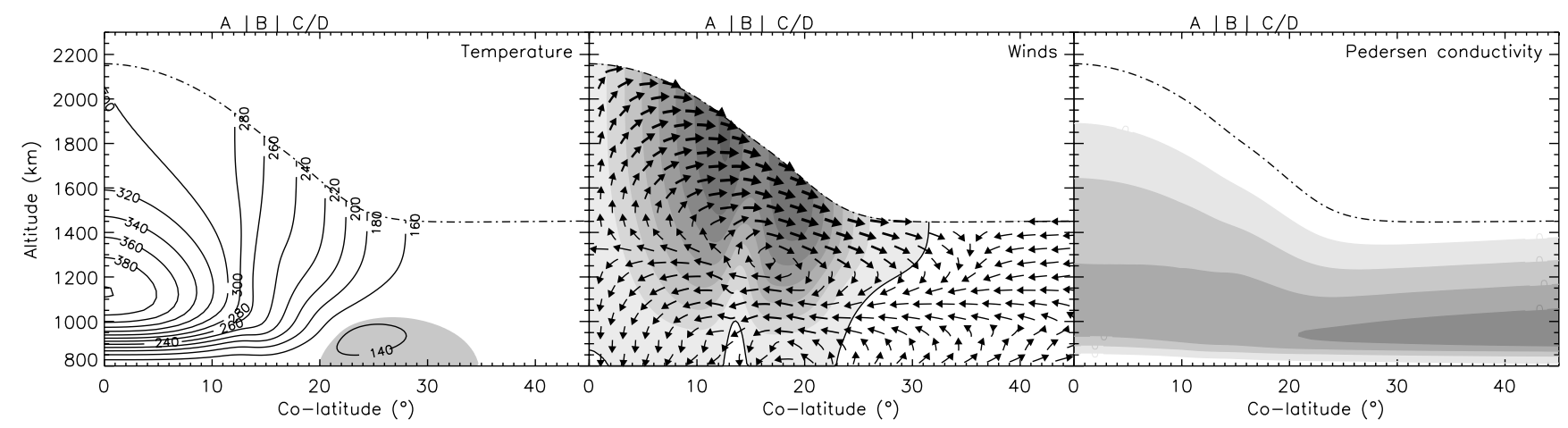

Fig. 9. Thermospheric response of modified model, in the same format as Fig. 5.

(a)

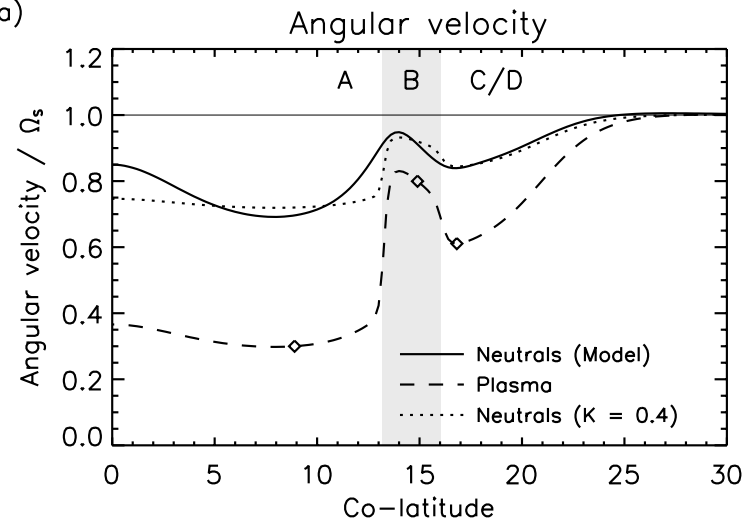

(c)

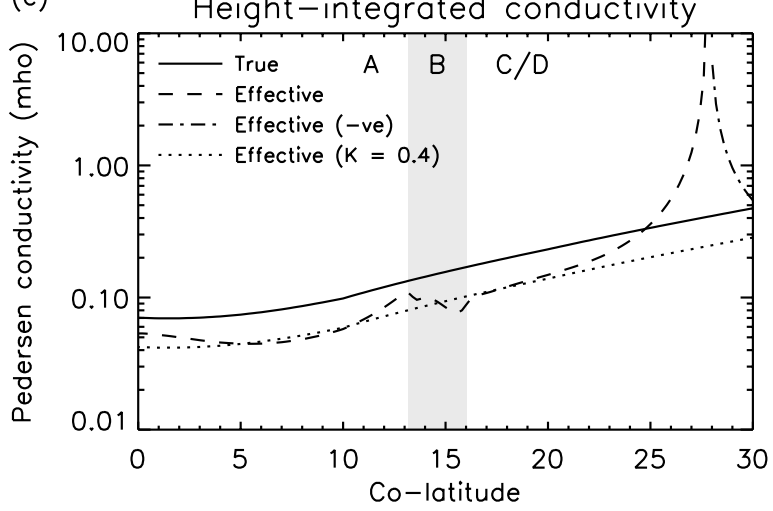

(b)

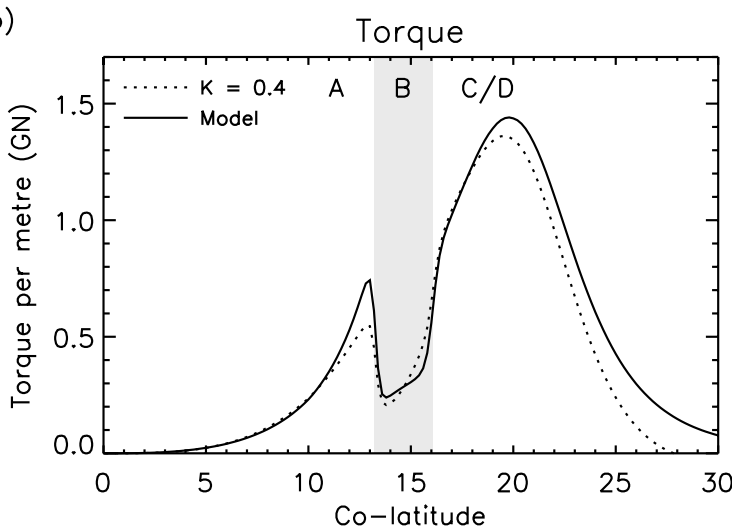

(d)

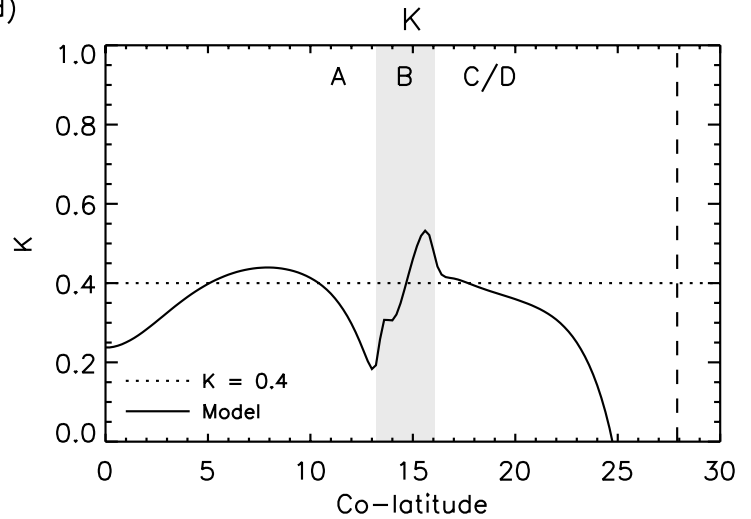

Fig. 10. Parameters for our modified magnetosphere model ( $\chi$-model) in the same format as Fig. 6 . The "anchor points" of the $\chi$-model are shown by the diamonds in panel (a). In panel (c), negative values of the effective conductivity are shown by plotting their absolute value as a dot-dash line. In panel (d) the vertical dashed line shows the point at which $K \rightarrow \infty$. To the left of this line $K$ is large and negative; to the right of this line $K$ is large and positive.

uniform physical properties in each region of the magnetosphere.

Importantly, the interaction between the thermosphere and magnetosphere is now a two way process. The constant- $K$ model implied that the rotational structure of the magneto- sphere was essentially imprinted on the thermosphere, which was a passive participant in the interaction. Now, the rotational structure of the magnetosphere is still imprinted on the thermosphere, but this structure is modified by the thermospheric neutral winds - which tend to smear out the structure 
and shift it slightly polewards - and the resulting thermospheric structure then feeds back into the rotational structure of the magnetosphere.

This new perspective suggests that thermospheric dynamics may influence the magnetospheric structure in more complicated ways than we are able to represent with our simple, steady state model. To illustrate this, let us consider a hypothetical situation in which a discrete episode of enhanced mass-loading occurs in the middle magnetosphere (region C). Such an episode would immediately begin to reduce the rotation of the plasma in this region of the magnetosphere, and thus to reduce the rotation of the connected neutrals via ion drag. This reduction in rotation velocity will be communicated to regions of the neutral atmosphere at higher latitudes by the prevailing polewards wind. This would then also cause the rotation velocity of the neutrals - and thus the plasma - in regions A and B to be reduced.

Meridional wind speeds in the conducting layer predicted by our model are typically in the range $10-100 \mathrm{~m} / \mathrm{s}$. At these velocities, changes in rotational velocity induced by the magnetosphere may be transported between 360-3600 km during every 10-h planetary rotation. One degree of latitude corresponds to $\sim 1000 \mathrm{~km}$. Thus changes in the rotational velocity of the inner magnetosphere may be communicated to thermospheric latitudes coupled to the outer magnetosphere within only a few planetary rotations. We thus suggest that the neutral atmosphere may mediate relatively short timescale interactions between regions of the magnetosphere that would otherwise be unconnected.

This role for the neutral atmosphere is not confined to the hypothetical discrete episode of mass loading outlined above. Our results imply that even if mass-loading of the magnetosphere is completely invariable, the neutral atmosphere has an important role to play in the radial transport of angular momentum, and its influence should be incorporated in any analysis of the magnetospheric angular momentum budget.

\subsubsection{Super-rotation}

The second motivation for our modified model was to allow us to represent super-rotation of the inner magnetosphere. As discussed in Sect. 3, super-rotation of the order of $\sim 5 \%$ is evident in the Voyager 2 data (Richardson, 1986) mapping to the range $20-25^{\circ}$ co-latitude, and a similar effect is present in the more recent Cassini data (Sittler et al., 2006).

In Fig. 10d, the existence of super-rotation is indicated by the behaviour of $K$. Negative values of $K$ at colatitudes greater than $25^{\circ}$ indicate that the neutral velocity is super-corotational, while the plasma velocity remains subcorotational; at $\sim 28^{\circ}$ co-latitude the vertical dashed line indicates that $K$ has changed from being strongly negative to being strongly positive. This region of strongly positive $K$ corresponds to a situation in which both the neutral and plasma velocities are super-corotational. Figure 11a shows a blowup of Fig. 10a in the region that exhibits super-rotation. It
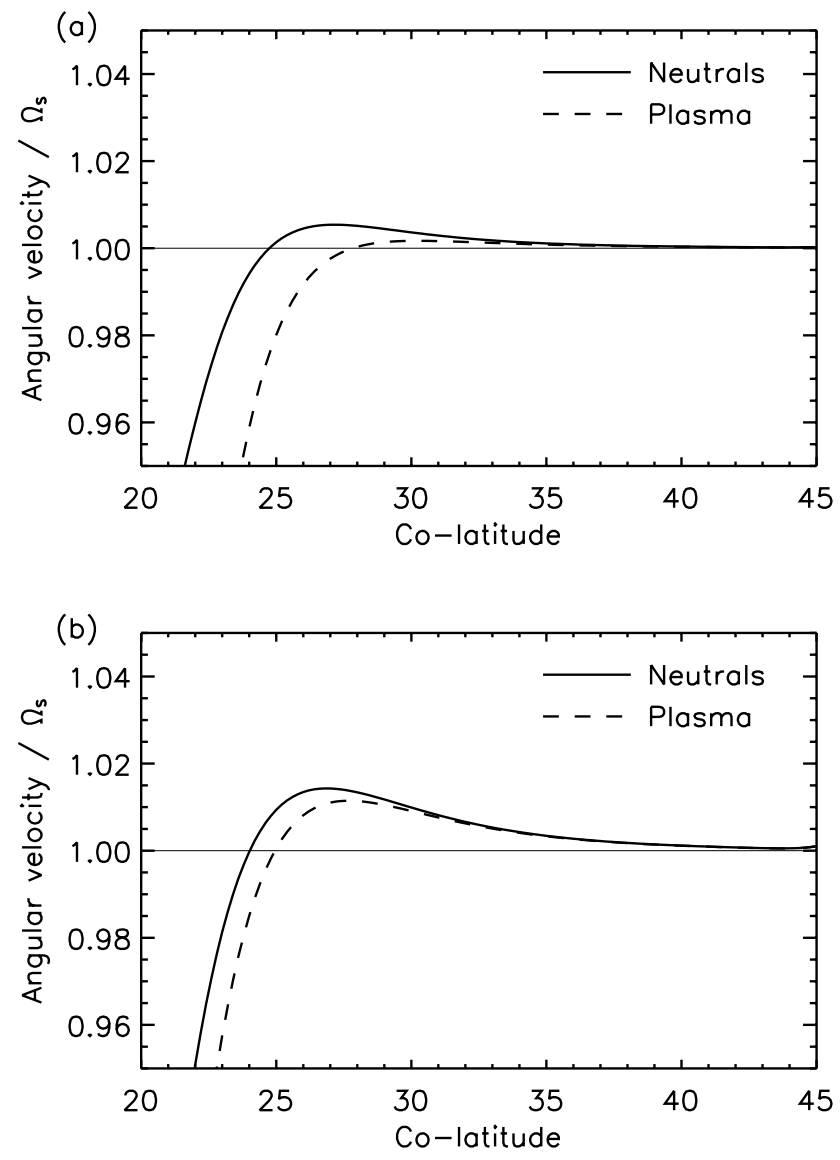

Fig. 11. Super-corotation of plasma mapping to the middle magnetosphere. (a) $\chi$-model, unscaled conductivity. (b) $\chi$-model, conductivity scaled up by a factor of 16 .

is clear that the degree of super-corotation is very small, of the order of less than $1 \%$ - much less than that observed by Voyager 2 - and that it lies at a greater co-latitude than the super-corotation seen in the data. However, the qualitative reproduction of this behaviour suggests that this flow signature may indeed be a result of the dynamics of the neutral atmosphere. We show in Sect. 7 below that larger quantitative effects can be produced in the model if the conductivity is increased.

\section{Sensitivity study}

Since the results presented above are not significantly altered by the introduction of the $\chi$-model it is not clear that it represents an essential modification rather than a somewhat cosmetic change. However, the model runs presented so far represent relatively mild conditions in terms of the magnitude of the ionospheric conductivity. As discussed by Smith et al. (2007), our conductivity model is most likely 
(a)
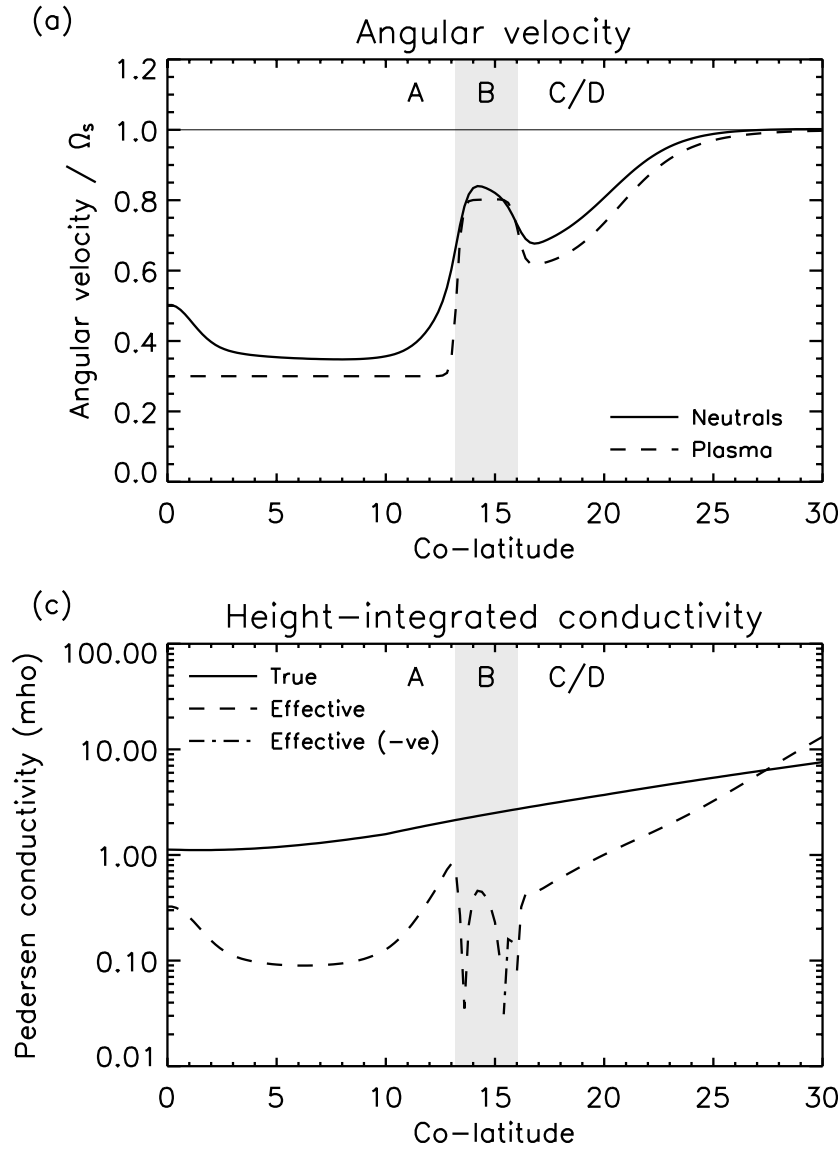

(b)

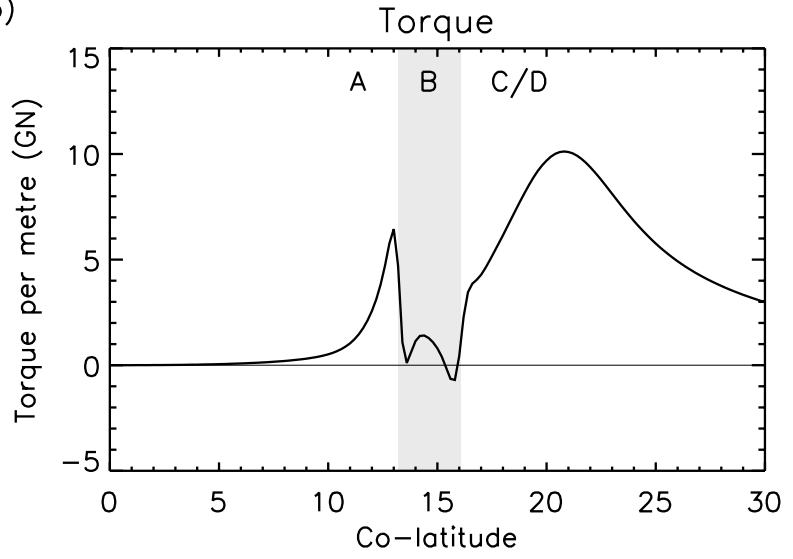

(d)

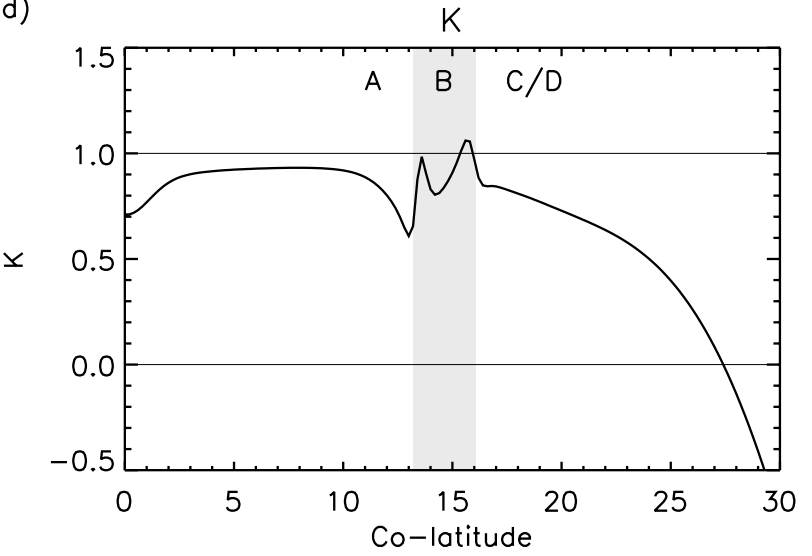

Fig. 12. Parameters for the $\Omega$-model with conductivities scaled up by factor of 16 , in the same format as Figs. 6 and 10 .

an underestimate of the real conductivity of the ionosphere. Smith et al. (2007) thus performed a sensitivity study in which they scaled up the conductivity model by a factor of 16. In Figs. 12 and 13 we show results for this increased conductivity model using the $\Omega$-model and $\chi$-model, respectively. The plasma velocity curve for the $\chi$-model is also shown in Fig. 2 by the dot-dashed line, demonstrating again that the results of the $\chi$-model are reasonably consistent with the data.

\subsection{Rotational structure}

Looking first at Fig. 12 - showing results from the unmodified, fixed $\Omega$-model - we can see similarly peculiar behaviour to that shown in Fig. 6. The most interesting aspect of this behaviour occurs just inside the equatorward edge of region B. Here the plasma velocity is slightly greater than the neutral velocity. This arises because the polewards flowing neutral gas is unable to spin up sufficiently quickly at the B-C boundary to keep up with the corresponding rise in the plasma velocity.
The consequence of this flow signature is that $K$ takes a value greater than unity, and both the torque and the effective conductivity become negative. Physically, this means that the magnetosphere is exerting a corotational torque on the thermosphere: a situation that does not make sense. The magnetosphere only rotates because it is driven into rotation by the thermosphere, so it is not physically reasonable for the magnetosphere to do the opposite and force the thermosphere towards corotation. This is of course a simplification - there may be circumstances in which radial flows in the magnetosphere force plasma to rotate more quickly than the connected neutral atmosphere - but in this case it is clearly an anomaly resulting from the fixed nature of the magnetosphere model, since we do not represent radial flows in our model.

Comparing these profiles to those in Fig. 13 - showing results from the $\chi$-model - it is clear that the $\chi$-model is again a significant improvement. The "wiggly" structures in the torque, effective conductivity, and $K$ disappear, and are replaced by reasonably simple and smooth variations. In particular, we no longer have a situation in which the torque is 
(a)

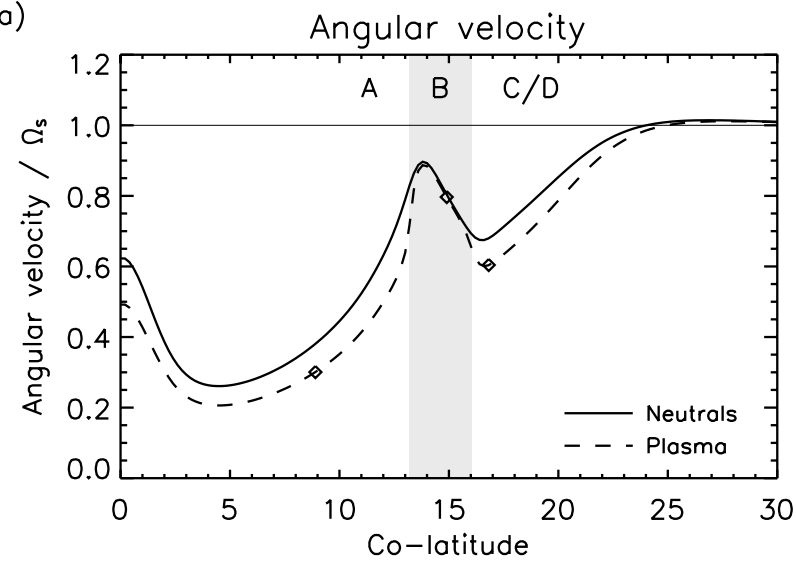

(c)

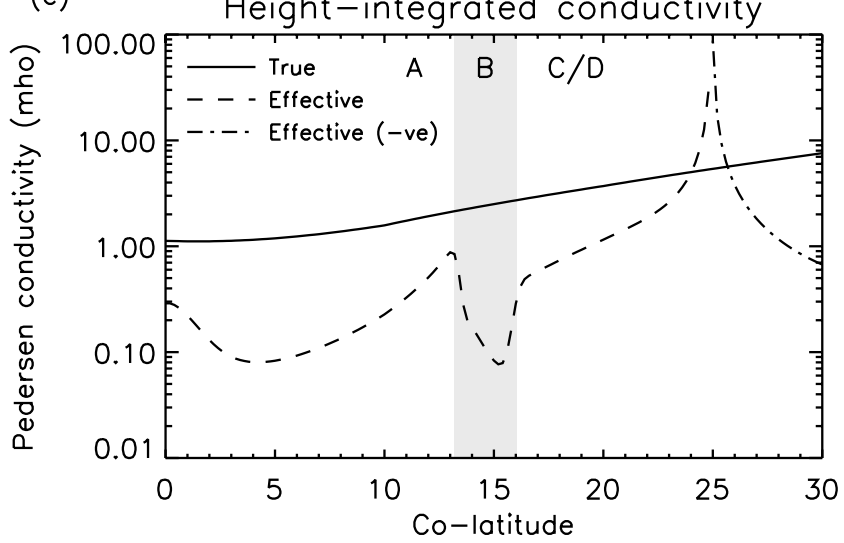

(b)

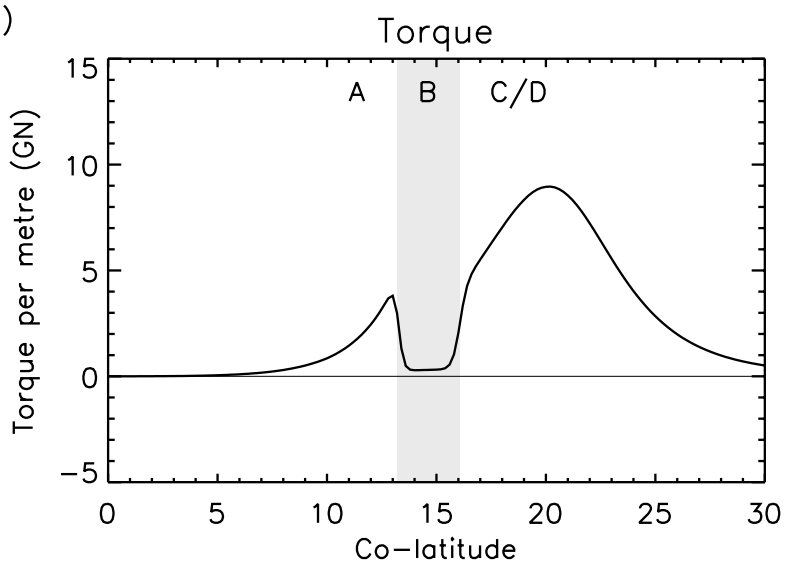

(d)

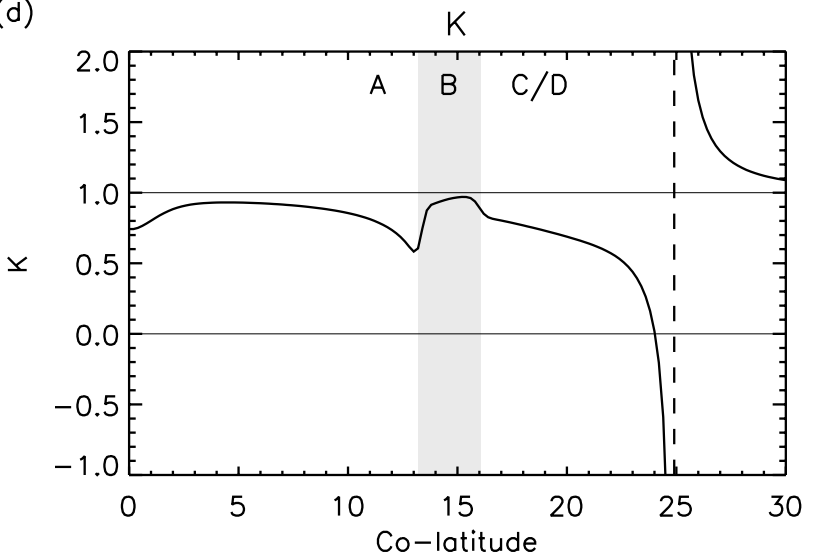

Fig. 13. Parameters for the $\chi$-model with conductivities scaled up by factor of 16, in the same format as Figs. 6 and 10.

negative and the magnetosphere is driving the thermosphere towards corotation.

Note that the $\chi$-model does not rule out the possibility of the magnetosphere rotating more quickly than the thermosphere. Since the value of $\chi$ for each region of the model is determined only by the requirement that a particular flow velocity is reproduced at each anchor point, it is perfectly possible for a value of $\chi>1$ to be required, corresponding to $\Omega_{M}>\Omega_{T}$. However, if this were to occur it could easily be identified as a pathological case and interpreted appropriately.

The flows predicted in region $\mathrm{B}$ of the $\chi$-model are also interesting. The plasma here subcorotates only marginally, such that the torque falls almost to zero. There is thus very little ion drag acting on the neutrals, and moving towards the pole the dominant effect is the Coriolis-driven spin-up of the neutrals. The flow velocities of both the plasma and the neutrals thus increases steadily across this region. This produces a very clear flow structure in the magnetosphere flow velocity increasing monotonically with increasing distance from the planet - that is driven by the behaviour of the neutrals. If there is, as suggested by Cowley et al. (2004), a region such as region $\mathrm{B}$ in the outer magnetosphere in which the torques acting between the thermosphere and magnetosphere are significantly reduced, we might expect to observe this flow structure - although we would certainly not expect it to be as clear cut as our model predicts.

Finally, the increase in the conductivity increases the degree of super-corotation observed in regions mapping to the middle magnetosphere. As shown in Fig. 11b, the degree of super-corotation exhibited by both the neutrals and the plasma increases to almost $2 \%$. This indicates that supercorotation comparable to that observed (5\%) is plausible within our model. Further work is required, though, to show whether it is possible to reproduce the observed degree of super-corotation at the correct latitude. In the long term, it may even be possible to use this flow signature as an observational constraint on the output of thermosphere models. 


\subsection{Thermal structure}

For the higher conductivity model we also find a significant difference in the thermal structure of the thermosphere between the $\Omega$ - and $\chi$-models. For the unscaled models the effect of the $\chi$-model was small - reducing the maxiumum temperature in the polar hotspot by $\sim 10 \mathrm{~K}$, about $\sim 2.5 \%$ of its absolute value of $\sim 400 \mathrm{~K}$. In the case of the higher conductivities the $\chi$-model reduces the maximum temperature in the polar hotspot by $\sim 60 \mathrm{~K}$, which corresponds to a $\sim 10 \%$ reduction of the absolute value of $\sim 550 \mathrm{~K}$. This reduction in temperature apparently arises because using the $\Omega$-model implies large plasma-neutral velocity differences close to the boundaries of flow regions - for example at the A-B boundary - which correspond to large quantities of Joule heating. This extra heating becomes incorporated in the polar hotspot. Thus using a fixed model can lead us to overestimate energy inputs to the thermosphere.

\section{Discussion}

As mentioned in Sect. 3, our initial, unscaled conductivity model exhibits true conductivities $\Sigma_{P}$ of order 0.1 mho, much lower than the effective conductivity $\Sigma_{P}^{*}=1 \mathrm{mho}$ adopted by Cowley et al. (2004) for use with their plasma flow model. Our scaled conductivity model exhibits a true conductivity of $\sim 1$ mho at the pole, increasing gradually to $\sim 7-8$ mho at $30^{\circ}$ co-latitude. However, looking at the effective conductivity that the model predicts (Fig. 13c), we can see that this is much lower than the true values, such that we only begin to reach values as high as 1 mho at colatitudes greater than $20^{\circ}$. This large discrepancy between the effective and true conductivities suggests that if the effective conductivity is to be of the order of $1 \mathrm{mho}$, then the true conductivity must be much larger than this. A proper assessment of how much larger the conductivity must be - and whether the ionospheric plasma densities required to generate this conductivity are consistent with the available data is beyond the scope of this study.

Our results do clearly indicate, though, that a single value of the effective conductivity is an inadequate parameterisation of the upper atmosphere. In Fig. 13c the effective conductivity at co-latitudes less than $25^{\circ}$ exhibits values ranging from less than 0.1 mho to greater than 10 mho. At colatitudes greater than $25^{\circ}$ it then becomes strongly negative due to the super-rotation of the plasma and neutrals. This means that blind application of a constant value of $\Sigma_{P}^{*}$ across the polar regions - especially when studying regions in which the plasma flow velocity varies rapidly - is likely to produce unreliable results.

It may be possible to employ the effective conductivity when only studying limited regions of the magnetosphere, since within regions of slowly varying plasma flow the effective conductivity also varies reasonably slowly. However, even in these cases the use of $\Sigma_{P}^{*}$ would still not be strictly valid, because its physical meaning really depends on the validity of the eddy viscosity model. This is because completely replacing $\Sigma_{P}$ with $\Sigma_{P}^{*}$ implies that the neutrals respond equivalently to all structures in the plasma flow, according to the linear behaviour predicted by the eddy viscosity model. Our results show that the response of the neutrals to a flow structure is dependent not only on that nature of that structure itself, but also on structures lying "upstream" from the perspective of the neutrals. Thus we think that the whole notion of an "effective conductivity" as a physically meaningful parameter is invalid.

It might still be argued that under circumstances not represented by our model the supply of angular momentum by eddy viscosity could become the dominant process. This might arise in two circumstances: firstly if there was no polewards flow to supply angular momentum, forcing it to be supplied by viscosity instead; secondly if the eddy coefficient is enhanced in the polar regions, increasing the rate of supply by that mechanism. We do not think that either of these is likely to be the case. As discussed by Smith et al. (2007), the polewards flow of gas that supplies angular momentum to the polar regions can be interpreted as driven by the atmosphere "collapsing" towards the pole to restore the hydrostatic equilbrium that has been perturbed by ion drag. The compressional heating due to the convergent flow at the pole partially restores this equilibrium by supporting an increased curvature of the upper atmosphere at the pole. The only way to "switch off" the polewards flow would thus be to supply extra thermal energy to the polar regions, such that the polewards flow of gas became unnecessary. However, if there were too much thermal energy supplied to the polar regions, the situation would be reversed, and the meridional flow would be towards the equator instead. Thus to switch off the meridional flow would require a very precise input of thermal energy that implied zero meridional flow. Unless there is some mechanism that might regulate such a situation, this seems extremely unlikely.

The second possibility - that the eddy coefficient is enhanced in the polar regions - is more difficult to assess. Firstly, we should comment that there is now no reason to invoke such an enhancement, since our new mechanism is apparently more than capable of supplying sufficient angular momentum to support partial corotation of the magnetosphere. However, supposing there was such an enhancement, its consequences would be complex. Firstly, it would raise the altitude of the homopause, such that hydrocarbons capable of chemically depleting the ionosphere would be mixed to higher altitudes. This would reduce ionospheric densities and conductivities, restricting the conducting layer to higher altitude regions where the neutral densities are lower. Secondly, it would also increase eddy conductive cooling of the polar regions. This might have the opposite effect to that intended - as discussed above, compressional heating of the polewards flow supplies thermal energy to the polar hotspot, 
so if conductive cooling of this region is increased the polewards flow might also increase in order to correspondingly increase the compressional heating and restore equilibrium. In summary, the consequences of an enhanced eddy coefficient are more complicated than a simple increase in the eddy viscosity, and a full assessment is beyond the scope of this study. However, even if angular momentum was supplied largely by eddy viscosity, it seems likely that meridional winds would still "smear" the neutral winds, producing many of the effects that we have noted.

If we are to discard the eddy viscosity model and the notion of an effective conductivity, how are we to simply parameterise the effects of the neutral atmosphere on the magnetosphere? It is clear that globally there is no simple parameterisation. However, for small latitude ranges within the thermosphere our model predicts that both the conductivity and the neutral rotation velocity are well-behaved and slowly varying. It thus may be valid to approximate the behaviour of small latitude ranges by a constant (or slowly varying) conductivity and a constant (or slowly varying) neutral rotation velocity. This means that there are two free parameters $-\Sigma_{P}$ and $\Omega_{T}-$ rather than the single free parameter $\Sigma_{P}^{*}$. Such a two-parameter model is clearly less convenient than the oneparameter effective conductivity model, but our study indicates that the former is more likely to be a realistic representation of the thermospheric behaviour.

While such a parameterisation may be valid for small latitude ranges, the behaviour close to large shears in the plasma flow velocity is much more complicated. It is obviously not practical for all studies of magnetospheric dynamics to use numerical thermosphere models such as that described here. It may thus be useful to build analytic or semi-analytic models of the neutral flow close to such boundaries to assess the behaviour of the neutrals without the computational restrictions of a full numerical model.

\section{Conclusions}

In this study we have used a numerical model of Saturn's thermosphere to study the coupling between the rotational dynamics of the thermosphere and magnetosphere. Our main conclusions are as follows:

1. Meridional advection is the principal mechanism by which angular momentum is supplied to the polar thermosphere. The conventional eddy viscosity model of Huang and Hill (1989) is thus invalid: we have shown their assumption that transport of angular momentum by meridional winds is negligible to be false. This conclusion is unambiguously the case for the upper atmosphere of Saturn under the conditions represented by our model: however, we have also argued that it seems very unlikely that conditions might exist in which transport by eddy viscosity dominates over transport by meridional advection.
2. As a result of this, we propose that the "effective Pedersen conductivity" is most likely a poor parameterisation of the upper atmospheric behaviour. Instead, we suggest that a two-parameter model using the effective neutral rotation velocity $\Omega_{T}$ and the true Pedersen conductivity $\Sigma_{P}$ may be a more accurate representation of the true thermospheric behaviour.

3. Advection of angular momentum by meridional winds tends to produce meridionally smoothed rotational structures in the thermosphere which feed back on the structure of the magnetosphere. This process may couple the rotational structures of the inner and outer magnetospheres on timescales of a few planetary rotations or less.

4. Super-rotation of plasma in the inner magnetosphere may arise due to super-rotation of the connected neutral atmosphere. This super-rotation is ultimately driven by the sub-corotation of the middle and outer magnetospheres.

There are a number of simple extensions to this study that will help to generalise our conclusions. Most important of these is the exploration of a number of different models of the ionospheric conductivity and investigating self-consistently the effect of an enhanced eddy coefficient. The study might also usefully be extended to Jupiter.

More complicated extensions to the model would also be useful. For example, we have not made any attempt to compare the torques that our model calculates with the actual torques exerted by the magnetosphere. Thus we cannot, with our present model, truly assess whether the flow supplies sufficient angular momentum to support the flows in the magnetosphere. To do this we would require a physical model of the magnetospheric rotation, rather than the empirical model that we have employed here.

\section{Appendix A}

\section{Details of plasma flow model}

The functions $T_{X Y}$ used to define the plasma velocity model are defined as follows:

$$
\begin{aligned}
& T_{A B}(F)=\frac{1}{2}\left(1+\tanh \frac{F-F_{A B}}{\Delta F_{A B}}\right) \\
& F_{A B}=1526 \mathrm{nTR}_{\mathrm{S}}^{2} \quad \Delta F_{A B}=50.0 \mathrm{nTR}_{\mathrm{S}}^{2} \\
& T_{B C}(F)=\frac{1}{1+\left(F_{B C} / F\right)^{n_{B C}}} \\
& F_{B C}=2200 \mathrm{nTR}_{\mathrm{S}}^{2} \quad n_{B C}=50.0 \\
& T_{C D}(F)=\frac{1}{1+\left(F_{C D} / F\right)^{n_{C D}}} \\
& F_{C D}=3600 \mathrm{nTR}_{\mathrm{S}}^{2} \quad n_{C D}=8.0
\end{aligned}
$$




\section{Appendix B}

\section{Ionospheric conductivities}

The following expressions are used to calculate the Pedersen and Hall conductivities $\sigma_{P i}$ and $\sigma_{H i}$ due to a particular ion $i$ :

$\sigma_{P i}=\frac{e n_{i}}{|B|} \frac{1}{r_{i}+r_{i}^{-1}}$

$\sigma_{H i}=\frac{e n_{i}}{|B|} \frac{r_{i}}{r_{i}+r_{i}^{-1}}$

where $e$ is the electronic charge, $B$ is the magnetic flux density, $n_{i}$ is the ion number density, and $r_{i}=v_{i n} / \Omega_{i}$ is the ratio of the ion-neutral collision frequency $v_{i n}$ to the ion gyrofrequency $\Omega_{i}$. We calculate $v_{i n}$ using the expression given by Banks and Kockarts (1973):

$v_{\text {in }}=2.6 \times 10^{-15} n_{n} \sqrt{\frac{\alpha_{0}}{\mu_{i n}}}$

where $\alpha_{0}$ is the polarisability of the neutral gas (in units of $10^{-30} \mathrm{~m}^{3}$ ), given as $0.82,0.667$ and 0.21 for $\mathrm{H}_{2}, \mathrm{H}$ and $\mathrm{He}$, respectively, $n_{n}$ is the number density of the neutral species in $\mathrm{m}^{-3}$, and $\mu_{i n}$ is the reduced mass of the neutral and ionised species in atomic mass units.

Acknowledgements. The simulations in this study were performed using the HiPerSPACE facility at UCL, funded by the UK Particle Physics and Astronomy Research Council (PPARC). C. G. A. Smith acknowledges receipt of a CASE studentship funded by PPARC and Sun Microsystems Ltd. The Voyager data shown in Fig. 2 was supplied by the NASA Planetary Data System; C. G. A. Smith thanks E. Bunce for help producing this figure.

Topical Editor I. A. Daglis thanks S. V. Badman for her help in evaluating this paper.

\section{References}

Achilleos, N., Miller, S., Tennyson, J., Aylward, A. D., MuellerWodarg, I., and Rees, D.: JIM: A time-dependent, threedimensional model of Jupiter's thermosphere and ionosphere, J. Geophys Res., 103, 20 089-20 112, 1998.

Atreya, S. K.: Eddy mixing coefficient on Saturn, Planet. Space Sci., 30, 849-854, 1982.

Banks, P. M. and Kockarts, G.: Aeronomy, Academic Press, 1973.

Bougher, S. W., Waite, J. H., Majeed, T., and Gladstone, G. R.: Jupiter Thermospheric General Circulation Model (JTGCM): Global structure and dynamics driven by auroral and Joule heating, J. Geophys. Res., 110, 4008, doi:10.1029/2003JE002230, 2005.

Cowley, S. W. H. and Bunce, E. J.: Corotation-driven magnetosphere-ionosphere coupling currents in Saturn's magnetosphere and their relation to the auroras, Ann. Geophys., 21, 1691-1707, 2003,

http://www.ann-geophys.net/21/1691/2003/.
Cowley, S. W. H., Bunce, E. J., and O'Rourke, J. M.: A simple quantitative model of plasma flows and currents in Saturn's polar ionosphere, J. Geophys Res., A18, 5212, doi:10.1029/2003JA010375, 2004.

Cowley, S. W. H., Wright, D. M., Bunce, E. J., Carter, A. C., Dougherty, M. K., Giampieri, G., Nichols, J. D., and Robinson, T. R.: Cassini observations of planetary-period magnetic field oscillations in Saturn's magnetosphere: Doppler shifts and phase motion, Geophys. Res. Lett., 33, 7104, doi:10.1029/2005GL025522, 2006.

Davis, L. J. and Smith, E. J.: A model of Saturn's magnetic field based on all available data, J. Geophys Res., 95, 15 257-15 261, 1990.

Dougherty, M. K., Achilleos, N., Andre, N., Arridge, C. S., Balogh, A., Bertucci, C., Burton, M. E., Cowley, S. W. H., Erdos, G., Giampieri, G., Glassmeier, K.-H., Khurana, K. K., Leisner, J., Neubauer, F. M., Russell, C. T., Smith, E. J., Southwood, D. J., and Tsurutani, B. T.: Cassini Magnetometer Observations During Saturn Orbit Insertion, Science, 307, 1266-1270, 2005.

Fuller-Rowell, T. J., Rees, D., Quegan, S., Moffett, R. J., Codrescu, M. V., and Millward, G. H.: A Coupled ThermosphereIonosphere Model (CTIM), in STEP Handbook of Ionospheric Models, pp. 217-238, SCOSTEP, 1996.

Gurnett, D. A., Kurth, W. S., Hospodarsky, G. B., Persoon, A. M., Averkamp, T. F., Cecconi, B., Lecacheux, A., Zarka, P., Canu, P., Cornilleau-Wehrlin, N., Galopeau, P., Roux, A., Harvey, C., Louarn, P., Bostrom, R., Gustafsson, G., Wahlund, J.-E., Desch, M. D., Farrell, W. M., Kaiser, M. L., Goetz, K., Kellogg, P. J., Fischer, G., Ladreiter, H.-P., Rucker, H., Alleyne, H., and Pedersen, A.: Radio and Plasma Wave Observations at Saturn from Cassini's Approach and First Orbit, Science, 307, 1255-1259, 2005.

Hill, T. W.: Inertial limit on corotation, J. Geophys Res., 84, 65546558, 1979.

Huang, T. S. and Hill, T. W.: Corotation lag of the Jovian atmosphere, ionosphere, and magnetosphere, J. Geophys Res., 94, 3761-3765, 1989.

Millward, G., Miller, S., Stallard, T., Achilleos, N., and Aylward, A. D.: On the dynamics of the jovian ionosphere and thermosphere IV: Ion-neutral Coupling, Icarus, 173, 200-211, 2005.

Moore, L. E., Mendillo, M., Müller-Wodarg, I. C. F., and Murr, D. L.: Modeling of global variations and ring shadowing in Saturn's ionosphere, Icarus, 172, 503-520, 2004.

Moses, J. I., Bézard, B., Lellouch, E., Gladstone, G. R., Feuchtgruber, H., and Allen, M.: Photochemistry of Saturn's Atmosphere. I. Hydrocarbon Chemistry and Comparisons with ISO Observations, Icarus, 143, 244-298, 2000.

Müller-Wodarg, I. C. F., Mendillo, M., Yelle, R. V., and Aylward, A. D.: A global circulation model of Saturn's thermosphere, Icarus, 180, 147-160, 2006.

Pontius, D. H.: Implications of variable mass loading in the Io torus: The Jovian flywheel, J. Geophys Res., 100, 19531-19540, 1995.

Pontius, D. H.: Radial mass transport and rotational dynamics, J. Geophys Res., 102, 7137-7150, 1997.

Richardson, J. D.: Thermal ions at Saturn - Plasma parameters and implications, J. Geophys Res., 91, 1381-1389, 1986.

Saur, J.: Turbulent Heating of Jupiter's Middle Magnetosphere, Astrophys. J., 602, L137-L140, 2004.

Saur, J., Mauk, B. H., Kaßner, A., and Neubauer, F. M.: A model 
for the azimuthal plasma velocity in Saturn's magnetosphere, J. Geophys Res., 109, 5217, doi:10.1029/2003JA010207, 2004.

Sittler, E. C., Thomsen, M., Johnson, R. E., Hartle, R. E., Burger, M., Chornay, D., Shappirio, M. D., Simpson, D., Smith, H. T., Coates, A. J., Rymer, A. M., McComas, D. J., Young, D. T., Reisenfeld, D., Dougherty, M., and Andre, N.: Cassini observations of Saturn's inner plasmasphere: Saturn orbit insertion results, Planet. Space Sci., 54, 1197-1210, 2006.

Smith, C. G. A.: Periodic modulation of gas giant magnetospheres by the neutral upper atmosphere, Ann. Geophys., 24, 2709-2717, 2006a,

http://www.ann-geophys.net/24/2709/2006/.

Smith, C. G. A.: Modelling the Thermospheres of the Giant Planets, Ph.D. thesis, UCL, 2006b.

Smith, C. G. A., Aylward, A. D., Miller, S., and Müller-Wodarg, I. C. F.: Polar heating in Saturn's thermosphere, Ann. Geophys., 23, 2465-2477, 2005,

http://www.ann-geophys.net/23/2465/2005/.
Smith, C. G. A., Miller, S., and Aylward, A. D.: Magnetospheric energy inputs into the upper atmospheres of the giant planets, Ann. Geophys., 23, 1943-1947, 2005, http://www.ann-geophys.net/23/1943/2005/.

Smith, C. G. A., Aylward, A. D., Millward, G., Miller, S., and Moore, L. E.: An unexpected cooling effect in Saturn's upper atmosphere, Nature, 445, 399-401, 2007.

Stallard, T. S., Miller, S., Trafton, L. M., Geballe, T. R., and Joseph, R. D.: Ion winds in Saturn's southern auroral/polar region, Icarus, 167, 204-211, 2004.

Vasyliūnas, V. M.: Plasma distribution and flow, pp. 395-453, Physics of the Jovian Magnetosphere, 1983. 